\title{
An Efficient and Stable Method for Computing Multiple Saddle Points with Symmetries
}

\author{
ZHI-QIANG WANG* and JiAnXIN ZHOU ${ }^{\dagger}$
}

\begin{abstract}
In this paper, an efficient and stable numerical algorithm for computing multiple saddle points with symmetries is developed by modifying the local minimax method established in $[12,13]$. First an invariant space is defined in a more general sense and a Principle of Invariant Criticality is proved for the generalization. Then the orthogonal projection operator to the invariant space is used both to preserve the invariance and to reduce computational error across iterations. Simple averaging formulas are used to find the orthogonal projection operators. Numerical computations of examples with various symmetries, of which some can and others cannot be characterized by a compact group of linear isomorphisms, are carried out to confirm the theory and to illustrate applications. The mathematical features of various symmetries demonstrated in these examples fall into two categories: nodal solutions of saddle point type with large Morse indices and non-radial positive solutions via symmetry breaking in radially symmetric elliptic problems. The new numerical algorithm generates these rather unstable solutions in a stable way. The existence of many unstable solutions and their behavior found in this paper remain to be investigated.
\end{abstract}

\section{Introduction}

Let $H$ be a Hilbert space and $J: H \rightarrow \mathbb{R}$ be Frechet differentiable, $J^{\prime}$ be its Frechet derivative and $\nabla J$ be the gradient and $J^{\prime \prime}$ its second Frechet derivative if it exists. A point $u^{*} \in H$ is a critical point of $J$ if $u^{*}$ solves the Euler-Lagrange equation $J^{\prime}\left(u^{*}\right)=0$. A critical point $u^{*}$ is

${ }^{*}$ Department of Mathematics and Statistics, Utah State University, Logan, UT 84322

${ }^{\dagger}$ Department of Mathematics, Texas A\& M University, College Station, TX 77843 
non-degenerate if $J^{\prime \prime}\left(u^{*}\right)$ is invertible, otherwise $u^{*}$ is degenerate. According to Morse theory, the Morse Index (MI) of a critical point $u^{*}$ of $J$ is the maximal dimension of a subspace of $H$ on which the operator $J^{\prime \prime}\left(u^{*}\right)$ is negative definite. The first candidates for a critical point are the local extrema to which the classical critical point theory was devoted in calculus of variations. Most conventional numerical algorithms focus on finding such stable solutions. Critical points that are not local extrema are unstable and called saddle points. Due to unstable nature, saddle points are very elusive to be numerically captured.

It is interesting for both theory and applications to develop efficient and stable numerical algorithms for finding multiple saddle points. Minimax principle is one of the most popular approaches in critical point theory. However, most minimax theorems in the literature (See [1], [18], [19], [20], [21], [24]), such as the mountain pass, various linking and saddle point theorems, require one to solve a two-level global optimization problem and therefore not useful for algorithm implementation.

Efforts for numerically computing saddle points have been made in [7] for $\mathrm{MI}=1$ and in [10] for $\mathrm{MI}=2$ which were motivated by theoretical (global minimax) characterizations of saddle points in [1] and [23] respectively. Inspired by [7, 10] and an idea in [9], a local minimax method (we shall refer it as LMM in the paper) was developed in $[12,13]$ and many multiple solutions were numerically computed for a class of semilinear elliptic equations. Its convergence results are obtained in [13]. Several results in instability analysis of saddle points are established in $[14,25]$.

We briefly recall LMM here. The basic idea of LMM is to define a local peak selection and a solution set. Let $L \subset H$ be a closed subspace, called a support to the critical point $u^{*}$ to be found, $S_{L^{\perp}}=\left\{v \in L^{\perp}:\|v\|=1\right\}$ and denote

$$
\{L, v\}=\left\{t v+v_{L}: t \in \mathbb{R}, v_{L} \in L\right\} \quad \forall v \in S_{L^{\perp}}
$$

A set-valued mapping $P: S_{L^{\perp}} \rightarrow 2^{H}$ is called a peak mapping of $J$ if $P(v)$ is the set of all local maximum points of $J$ in $\{L, v\}$. A single-valued mapping $p: S_{L^{\perp}} \rightarrow H$ is called a peak selection if $p(v) \in P(v) \forall v \in S_{L^{\perp}}$. Let $v \in S_{L^{\perp}}$ be a point. $p$ is said to be a local peak selection of $J$ w.r.t. $L$ at $v$ if there exists a neighborhood $\mathcal{N}(v)$ of $v$ and $p$ is a locally defined mapping $p: \mathcal{N}(v) \cap S_{L^{\perp}} \rightarrow H$ s.t. $p(u) \in P(u) \forall u \in \mathcal{N}(v) \cap S_{L^{\perp}}$.

A local minimax theorem which characterizes a saddle point as a local minimax solution has been established in [12]. It states that let

$$
\mathcal{M}=\left\{p(v): v \in S_{L^{\perp}}\right\}
$$


be a solution set, a point $u^{*}=p\left(v^{*}\right) \in \mathcal{M}$ is a saddle point if

$$
v^{*}=\arg \min _{v \in S_{L^{\perp}}} J(p(v))=\arg \min _{v \in S_{L^{\perp}}} \max _{u \in\{L, v\}} J(u) .
$$

It becomes a local minimization problem on the solution set $\mathcal{M}$, which can be numerically approximated by, e.g., the steepest descent method.

\section{A Numerical Local Min-max Method}

Step 1: Given positive $\varepsilon$ and $\lambda$. Let $n-1$ critical points $w_{1}, w_{2}, \ldots, w_{n-1}$ of $J$ be given, of which $w_{n-1}$ has the highest critical value. Set the support $L=\operatorname{span}\left\{w_{1}, w_{2}, \ldots, w_{n-1}\right\}$. Let $v_{n}^{1} \in S_{L^{\perp}}$ be an ascent direction at $w_{n-1}$. Let $t_{0}^{0}=1$ and $v_{L}^{0}=w_{n-1}$ and set $k=0$;

Step 2: Use the initial guess $w=t_{0}^{k} v^{k}+v_{L}^{k}$, solve for

$$
w^{k} \equiv p\left(v^{k}\right) \equiv t_{0}^{k} v^{k}+v_{L}^{k}=\arg \max _{w \in\left\{L, v^{k}\right\}} J(w) \quad \text { and denote } \quad t_{0}^{k} v^{k}+v_{L}^{k}=w^{k} \equiv p\left(v^{k}\right)
$$

Step 3: Compute the steepest descent vector $d^{k}=-\nabla J\left(w^{k}\right)$;

Step 4: If $\left\|d^{k}\right\| \leq \varepsilon$ then output $w_{n}=w^{k}$, stop; else goto Step 5;

Step 5: Set

$$
v^{k}(s)=\frac{v^{k}+s d^{k}}{\left\|v^{k}+s d^{k}\right\|}
$$

and find

$$
s^{k}=\max \left\{\frac{\lambda}{2^{m}} \mid m \in \mathbb{N}, J\left(p\left(v^{k}\left(\frac{\lambda}{2^{m}}\right)\right)\right)-J\left(w^{k}\right) \leq-\frac{t_{0}^{*}}{2}\left\|d^{k}\right\|\left\|v^{k}\left(\frac{\lambda}{2^{m}}\right)-v^{k}\right\|\right\} .
$$

Initial guess $u=t_{0}^{k} v^{k}\left(\frac{\lambda}{2^{m}}\right)+v_{L}^{k}$, where $t_{0}^{k}$ and $v_{L}^{k}$ are found in Step 2, is used to find the local maximum point $p\left(v^{k}\left(\frac{\lambda}{2^{m}}\right)\right)$ in $\left\{L, v^{k}\left(\frac{\lambda}{2^{m}}\right)\right\}$ as similar as in Step 2 .

Step 6: Set $v^{k+1}=v^{k}\left(s^{k}\right)$ and update $k=k+1$ then goto Step 2.

The subspace $L$ containing critical points previously found serves as a support to a saddle point $u^{*}$ to be found at a higher critical level. A support to $u^{*}$ is said to be sufficient if it contains all critical points below $u^{*}$ 's critical level. When the Morse index of $u^{*}$ gets larger, the dimension of $L$ grows larger [25]. Solving the local maximization problem in the space $\left\{L, v^{k}\right\}$ in Step 2 of LMM becomes more expensive. Since $u^{*}=p\left(v^{*}\right)$ and $v^{*}=\arg \max _{v \in S_{L^{\perp}}} J(p(v))$, the solution set $\mathcal{M}$ is a stable set, i.e., when $J$ is restricted to $\mathcal{M}, u^{*}$ is a stable solution. 
Symmetries exist in many natural phenomena, such as in crystals, elementary particle physics, symmetry of the Schrodinger equation for the atomic nucleus and the electron shell w.r.t. permutations and rotations, energy conservation law for systems which are invariant w.r.t. time translation, etc. Symmetry properties are usually studied by group actions in mathematics. Symmetries described by compact group actions in variational problems have been used in the literature to prove the existence of multiple critical points, typically, in the Ljusternik-Schnirelman theory (see recent results in $[15,11]$ and others). It is known that symmetries in a nonlinear variational problem can lead to the existence of more solutions of saddle type and can also cause degeneracy. In this paper, we study the impact of the presence of symmetries on LMM in finding multiple saddle points. By modifying LMM, we shall develop an efficient and stable numerical algorithm for computing multiple critical points with general symmetries. Consider a semilinear elliptic BVP

$$
\begin{cases}\Delta u(x)+f(u(x), x)=0, & x \in \Omega \subset R^{n}, \\ u(x)=0, & x \in \partial \Omega\end{cases}
$$

where $f$ satisfies some standard conditions. When $L=\{0\}$, the solution set

$$
\mathcal{M}=\left\{t_{u} u: u \in H,\|u\|=1, t_{u}>0, \nabla J\left(t_{u} u\right) \perp u\right\}
$$

is called the Nehari manifold. Under some standard conditions, it can be shown that $\mathcal{M}$ is smooth, or the peak selection $p$ is continuously differentiable [12].

Several researchers have tried to use certain symmetry of a problem to capture a solution of higher Morse index. For example, odd symmetry is used in [7] to capture sign-changing solutions $(\mathrm{MI}=2)$ by a minimization on the Nehari manifold with an odd symmetric initial guess. When a negative gradient-type minimax algorithm is used, the symmetry is inherited but not enforced, the sequence generated by the algorithm will get close to a saddle point. However, when computational error builds up or $\|\nabla J(u)\|$ becomes small, computational error will dominate and break the symmetry of $\nabla J(u)$. Eventually the symmetry of the sequence collapses. Therefore, the sequence will not stop near a sign-changing solution, instead it will slide down to a positive solution $(\mathrm{MI}=1)$, unless a forcing stop action is taken. Thus such an approximation is unstable and no convergence can be established. Even rotational symmetry is considered in [8] to capture sign-changing solutions $(\mathrm{MI}=3)$ by a high-linking algorithm. Action to preserve the symmetry is taken, so the algorithm is stable. 
In this paper, we consider more general symmetries and try to establish some mathematical justifications. In particular, we are concerned with not only preserving the symmetry but also reducing computational error across iterations.

There are at least three motivations for one to use symmetries to define an invariant space $H_{I}$ in computing a saddle point,

(1) a sufficient support is available in $H$ but one wants to reduce its dimension by using a sufficient support $L$ in $H_{I}$ to enhance the efficiency and convergence of the algorithm;

(2) no sufficient support is available in $H$, one has to use the symmetries to find a sufficient support $L$ in $H_{I}$;

(3) to use symmetries to bypass the degeneracy of a problem.

To find a critical point $u^{*}$ at a higher critical level by LMM, one needs to know if the support $L$ is sufficient or not. If the answer is yes, one can expect a stable convergence even without using any symmetry. If the answer is no, the minimization process will sooner or later find a slider and bypass $u^{*}$. The algorithm becomes unstable and fails to reach $u^{*}$.

Assume one has identified symmetries of a problem and defined an invariant subspace $H_{I}$. Then one can restrict the problem in $H_{I}$, i.e., the support $L$ contains critical points at lower critical level only in $H_{I}$. By doing so, the dimension of the support $L$ can be greatly reduced. Since LMM with an insufficient support in $H_{I}$ is unstable, we assume the support $L$ in $H_{I}$ is sufficient. When computational error builds up or $\left\|\nabla J\left(w^{k}\right)\right\|$ becomes small, computational error will dominate and break the symmetry of $\nabla J\left(w^{k}\right)$. That is, $\nabla J\left(w^{k}\right)$, eventually $w^{k+1}$ goes to outside of $H_{I}$. There are two possibilities. If the support $L$ in $H_{I}$ is also sufficient in $H$, there is no slider around $u^{*}$. Thus the symmetry has no effect on the algorithm and therefore the collapse of the symmetry has no effect either, the algorithm will still converge to $u^{*}$; If the support $L$ in $H_{I}$ is not sufficient in $H$, and $w^{k+1}$ is outside $H_{I}$, the minimization process will sooner or later find a slider and then fail to reach $u^{*}$. A projection of $\nabla J\left(w^{k}\right)$ onto $H_{I}$ will pull $\nabla J\left(w^{k}\right)$ and then $w^{k+1}$ back to $H_{I}$ and resolve the problem.

These type of projections into an invariant space have been used in the literature to preserve the symmetry, where computational error is not a concern. In this case any projection operator onto $H_{I}$ will serve the purpose. However, computational error is a main concern in numerical computation, in particular, in multi-level iterations for finding unstable saddle points at a higher critical level, which is rather sensitive to computational error. It is therefore a main 
concern of this paper. There are infinitely many projection operators onto $H_{I}$. Some of them are poor to handle computational error. Only the orthogonal projection operator onto $H_{I}$ is the optimal one to handle computational error. Thus in this paper, we look for the orthogonal projection operator onto $H_{I}$. It is known that finding an orthogonal projection onto a subspace is equivalent to an infinite-dimensional minimization problem which is very expansive. It is in particular, very difficult, since there is no explicit expression for $H_{I}$. The average formula defined by the Haar integral has been used in the literature to project a point onto an invariant subspace. Here we expose a fact that the Haar integral operator is actually the orthogonal projection operator onto $H_{I}$. Implementation of this formula with LMM for numerical computations of multiple critical points with symmetries at higher critical levels will be discussed in detail by using typical numerical examples.

The numerical examples we choose also serve revealing new phenomena in the corresponding mathematical problems. Here we are mainly interested in two directions: nodal solutions of saddle point type for nonlinear elliptic problems and non-radial positive solutions in radially symmetric elliptic equations. In both cases, people expect that many unstable solutions exist, these solutions should have large Morse indices, and that degeneracy occurs in general. Using LMM with symmetry we shall not only demonstrate solutions that are known to exist in theory but also exhibit many cases for which the existence is still open in theory. Some of these examples give surprising, new mathematical features and should shed lights to the study of the nonlinear elliptic PDEs.

\section{Invariant Space and its Orthogonal Projection}

\subsection{Invariant Spaces and LMM in Invariant Spaces}

In order to let LMM handle symmetry we need the concept of invariant spaces. The following is a more general one without reference to a symmetry.

Definition 2.1 Let $H$ be a Hilbert space and $J \in C^{1}(H, R)$. A closed subspace $H_{I}$ of $H$ is said to be a $J$-invariant space if for every $u \in H_{I}$ it holds $\nabla J(u) \in H_{I}$.

It is clear that $H$ is trivially a $J$-invariant space. Since, in general, the smaller is an invariant space $H_{I}$, the smaller is the Morse index of $u^{*}$ relative to $H_{I}$ which implies that the smaller 
is the dimension of the support $L$ [25], and therefore, the more efficient is the numerical computation, we always look for the smallest such $J$-invariant space.

Along the line of the classical Principle of Symmetric Criticality (PSC) by Palais, we have the following Principle of Invariant Criticality (PIC) without reference of a symmetry.

Theorem 2.1 Let $H$ be a Hilbert space, $J \in C^{1}(H, R)$ and $H_{I}$ be a J-invariant space. If $u^{*} \in H_{I}$ is a critical point of $J$ restricted to $H_{I}$, then $u^{*}$ is a critical point of $J$ in $H$.

Proof: $u^{*}$ is a critical point of $J$ restricted to $H_{I}$ implies $\left\langle\nabla J\left(u^{*}\right), v\right\rangle=0$ for all $v \in H_{I}$, i.e., $\nabla J\left(u^{*}\right) \perp H_{I}$. On the other hand, $H_{I}$ is a $J$-invariant space, i.e., $u^{*} \in H_{I}$ implies $\nabla J\left(u^{*}\right) \in H_{I}$. Therefore $\nabla J\left(u^{*}\right) \in H_{I} \cap H_{I}^{\perp}=\{0\}$.

Assume a $J$-invariant space $H_{I}$ has been identified. Since $H_{I}$ is usually much smaller than $H$, replacing $H$ by $H_{I}$, LMM can be used, in a much more efficient and stable way, to find multiple saddle points with certain symmetries at higher critical level.

If $L$ is a closed subspace of $H_{I}$, a support to a critical point $u^{*}$ to be found in $H_{I}$ and denote $S_{L^{\perp}}=\left\{v \in H_{I}:\|v\|=1, v \perp L\right\}$. Let $k=0$, started from a point $v^{k} \in S_{L^{\perp}}$, we have $p\left(v^{k}\right)=t^{k} v^{k}+v_{L}^{k} \in H_{I}$ for some $t^{k} \neq 0$ and $v_{L}^{k} \in L \subset H_{I}$. Then $d^{k}=-\nabla J\left(p\left(v^{k}\right)\right) \in H_{I}$ and $v^{k+1}=\frac{v^{k}+s^{k} d^{k}}{\left\|v^{k}+s^{k} d^{k}\right\|} \in H_{I}$ where $s^{k}>0$ is the stepsize. If $v^{0} \in H_{I}$ and $d^{k}=-\nabla J\left(p\left(v^{k}\right)\right) \neq 0$, we have $[12]$

$$
J\left(p\left(v^{k+1}\right)\right)-J\left(p\left(v^{k}\right)\right)<-\frac{t^{k}}{2}\left\|d^{k}\right\|\left\|v^{k+1}-v^{k}\right\| .
$$

It concludes that, in theory, LMM is closed in an invariant space $H_{I}$ and generates a strict minimizing sequence $\left\{p\left(v^{k}\right)\right\}=\left\{t^{k} v^{k}+v_{L}^{k}\right\}$ in $\mathcal{M} \cap H_{I}$ where $\mathcal{M}$ is the solution set defined in (1.1). The limit of the sequence is a critical point of $J$ in $H_{I}$ by the convergence results in [13] and thus a critical point of $J$ in $H$ by PIC. However, in numerical computation of the negative gradient $d^{k}=-\nabla J\left(p\left(v^{k}\right)\right)$, it involves discretization, approximation, round-off error, etc. It generates numerical error and then breaks the invariance of $d^{k}$. To preserve the invariance, we use the decomposition $H=H_{I} \oplus H_{I}^{c}$ for some complement space $H_{I}^{c}$ of $H_{I}$ in $H$ and

$$
d^{k}=d_{I}^{k}+\left(d^{k}\right)_{I}^{c}, \quad d_{I}^{k} \in H_{I},\left(d^{k}\right)_{I}^{c} \in H_{I}^{c}
$$

Note that if $p\left(v^{k}\right) \in H_{I}$ and $d^{k}=-\nabla J\left(p\left(v^{k}\right)\right)$ is computed exactly, we should have

$$
d^{k}=\left(d^{k}\right)_{I} \in H_{I} \quad \text { and } \quad\left(d^{k}\right)_{I}^{c}=0
$$

When numerical error is involved, we use $\left(d^{k}\right)_{I}$ to replace $d^{k}$ in Step 3 of LMM, the updated point $v^{k+1}$ is now in $H_{I}$ and the invariance is preserved. 
The above decomposition for $H$ needs to find a projection operator $T$ from $H$ to $H_{I}$. There are infinitely many such projection operators. If preserving the invariance is the only concern, by PIC, any one of them will serve the purpose. However, when error analysis is concerned, the case is different. Note that the term $\left(d^{k}\right)_{I}^{c}=d^{k}-\left(d^{k}\right)_{I}$ represents computational error. In numerical computation, we have not only to preserve the invariance, but also to reduce error which will be carried to the next iteration. Among all those projection operators, some of them are very poor to deal with error and there is the optimal one that minimizes the distance from $d^{k}$ to $H_{I}$, the error term, that is the orthogonal projection. In this case, the maximum invariant part of $d^{k}$ has been carried to $\left(d^{k}\right)_{I}$ and $\left(d^{k}\right)_{I}^{c}$ becomes $\left(d^{k}\right)_{I}^{\perp}$, i.e.,

$$
d^{k}=\left(d^{k}\right)_{I}+\left(d^{k}\right)_{I}^{\perp} \quad \text { and } \quad\left(d^{k}\right)_{I} \perp\left(d^{k}\right)_{I}^{\perp}
$$

For this reason, in this paper we will do our best to adopt the orthogonal projection.

Example 2.1 Consider $X=R^{2}, X_{2}=\left\{\left(0, x_{2}\right)^{T}\right\}$. Both

$$
A=\left[\begin{array}{cc}
0 & 0 \\
100 & 1
\end{array}\right] \quad \text { and } B=\left[\begin{array}{ll}
0 & 0 \\
0 & 1
\end{array}\right]
$$

are projection operators of $X$ onto $X_{2}$. Let $\left(\epsilon_{1}, \epsilon_{2}\right)^{T}$ represents computational error in computing $(0,1)^{T} \in X_{2}$, we get $u=\left(\epsilon_{1}, \epsilon_{2}+1\right)^{T} \notin X_{2}$. To do projection, we have

$$
A u=\left(0,100 \epsilon_{1}+1+\epsilon_{2}\right)^{T} \in X_{2} \quad \text { and } \quad B u=\left(0,1+\epsilon_{2}\right)^{T} \in X_{2} .
$$

It is clear that $A$ greatly enlarges error while $B$ does not. As a matter of fact, when error analysis is concerned, $B$ is the optimal operator that minimizes error, i.e., $B$ is the orthogonal projection operator from $X \rightarrow X_{2}$ and $B u$ is the best approximation one can get.

\subsection{Invariant Spaces from Symmetries}

Invariant spaces appear naturally when the problems considered possess certain symmetry, e.g., when the functional $J$ is invariant with respect to certain symmetry. There are two usual ways in numerical computations to preserve a symmetry. By dividing the domain into several sub-domains along the axes of symmetry, one may solve the problem only on one sub-domain with an additional continuity condition with the Neumann data across the cuts, the problem may become much harder to solve, but the size of the problem becomes smaller; 
one may also solve the problem on the entire domain, but use the solution data only on one sub-domain. Then in either way, one can produce a solution on the entire domain according to the symmetry. Note that in either way, it preserves the symmetry, but carries computational error with the solution to the next iteration. Indeed it forces computational error to be of the same symmetry. However, it is known that computation error is usually asymmetric and even random.

Contrast to the usual method, our new method separates a solution from computation error (at least the asymmetric part), and then carries only the solution, not the error, in iterations. Thus the advantage is clear that it makes the algorithm more efficient and more stable. Our numerical examples confirm the analysis.

Let us start with the most studied symmetries in the literature, i.e., those symmetries that can be characterized by a compact group of linear isomorphisms.

Let $H$ be a Hilbert space and $G$ be a compact group of linear isomorphisms of $H$, i.e., the map from $G \times H \rightarrow H$ evaluated by $(g, u) \rightarrow g u$ is continuous such that

$$
1 \cdot u=u, \quad(g v) u=g(v u), \quad u \rightarrow g u \quad \text { is linear, } \quad\|g u\|=\|u\| .
$$

A set $A \subset H$ is G-invariant if $g(A)=A$ for every $g \in G$. The subspace

$$
H_{G}=\{u \in H: g u=u, \forall g \in G\}
$$

is called the invariant subspace of $H$ under $G$. Let $J \in C^{1}(H, R)$. $J$ is said to be G-invariant if $J(g u)=J(u)$ for every $(g, u) \in G \times H$. A map $F: H \rightarrow H$ is G-equivariant if $g \circ F=F \circ g$ for every $g \in G$. Since $J \in C^{1}(H, R)$ is G-invariant implies that $\nabla J$ is G-equivariant, i.e., $\nabla J(g u)=g \nabla J(u) \forall u \in H$. When $u \in H_{G}$, we have $g \nabla J(u)=\nabla J(g u)=\nabla J(u) \forall g \in$ $G \quad$ or $\quad \nabla J(u)=\nabla J(u)_{G}$.

It is clear that the above definition for an invariant space separates the space $H$ from the functional $J$, which may have other applications. On the other hand, Definition 2.1 combines the space $H$ with the functional $J$ in the definition of a $J$-invariant space, which serves precisely the purpose of applications in this research. It is clear that if $J$ is $G$-invariant, then $H_{G}$ is a $J$-invariant space as in Definition 2.1. Thus the following classical result follows from Theorem 2.1.

Theorem 2.2 (Principle of symmetric criticality, Palais, 1979) Let $H$ be a Hilbert space and $G$ be a compact group of linear isomorphisms of $H$. If $J \in C^{1}(H, R)$ is $G$-invariant and if $u \in H_{G}$ is a critical point of $J$ restricted to $H_{G}$, then $u$ is a critical point of $J$. 


\subsection{Orthogonal Projections}

Note that the invariant space $H_{G}$ is usually infinite-dimensional, finding the orthogonal projection of $d^{k}$ onto $H_{G}$ is equivalent to solving an infinite-dimensional optimization problem. It is very expensive. It is also very difficult since we do not have an explicit expression for $H_{G}$. However, for many usual symmetries, simple algebraic computations can be used to find the orthogonal projection onto $H_{G}$.

In order to preserve the invariance and minimize computational error across iterations, we need to construct the orthogonal projection operator from $H$ to $H_{G}$. Let us first cite

Theorem 2.3 (Haar, 1933) Let $G$ be a compact group and $C(G)$ be the vector space of realvalued continuous functions on $G$. Then there exists a unique positive integral (the Haar integral) such that the map $: C(G) \rightarrow R$ by $f \mapsto \int_{G} f(g) d g$ is

(a) linear, monotone and normalized $\left(\int_{G} 1 d g=1\right)$;

(b) left-invariant, i.e., $\int_{G} f(h g) d g=\int_{G} f(g) d g, \forall h \in G$.

The Haar integral defines the normalized left-invariant Haar integral operator $\mathcal{H}$ from $H$ to $H_{G}$ by

$$
\mathcal{H} u=\int_{G} g u d g, \quad \forall u \in H .
$$

The Haar operator has been used as an projection from $H$ onto $H_{G}$ in the literature to preserve an invariance, where computational error is not a concern. When reducing computational error across iterations is concerned, we are interested mainly in the orthogonal projection operator onto $H_{G}$. Since for $u, v \in H$,

$$
\langle\mathcal{H} u, \mathcal{H} v\rangle=\int_{G}\langle g u, \mathcal{H} v\rangle d g=\int_{G}\left\langle u, g^{*} \mathcal{H} v\right\rangle d g=\int_{G}\langle u, \mathcal{H} v\rangle d g=\langle u, \mathcal{H} v\rangle
$$

we have

$$
\langle u-\mathcal{H} u, v\rangle=\langle u-\mathcal{H} u, \mathcal{H} v\rangle=0 \quad \forall v \in H_{G},
$$

i.e., $\mathcal{H}$ turns out to be the orthogonal projection operator from $H$ onto $H_{G}$ and $u=\mathcal{H} u+$ $(u-\mathcal{H} u)$ is the orthogonal direct sum.

\subsection{Examples}

We give some examples that will be used in our numerical computations. 
Example 2.2 Let $H$ be a Hilbert space with inner product $\langle$,$\rangle and G$ be a finite group of linear isomorphisms of $H$ with $m$ elements. Then for each $u \in H$, the Haar integral operator on $u$ is given by

$$
\mathcal{H} u=u_{G}=\frac{1}{m} \sum_{g \in G} g u \in H_{G} \quad \text { and } \quad u_{G}^{\perp}=u-u_{G} \in H_{G}^{\perp} .
$$

Example 2.3 Let $\Omega \subset R^{n},(n \geq 1)$ be a bounded open set and $H=W^{k, 2}(\Omega)$ where $k \geq 0$ be the Sobolev space. Let $\mathcal{O}(n)$ be the set of all orthogonal matrices in $R^{n \times n}$. Then it is a compact group. Let $G$ be the set of all orthogonal matrices $g \in \mathcal{O}(n)$ such that $g(\Omega)=\Omega$. For each $g \in G$ and $u \in H$, if we define

$$
g u(x)=u(g x), \quad \forall x \in \Omega,
$$

then $G$ is a compact group of linear isomorphisms of $H$. The Haar integral operator $\mathcal{H}$ defines the orthogonal projection operator from $H$ onto $H_{G}$. Indeed, if $g$ is represented by an orthogonal matrix, then $g$ is an isomorphism of $H$ or the inner product is $g$-invariant, as we have for all $u, v \in H$,

$$
\begin{aligned}
\langle g u, g v\rangle= & \int_{\Omega} \sum_{|\alpha| \leq k}\left(D^{\alpha} u(g x)\right)^{T}\left(D^{\alpha} v(g x)\right) d x \\
& \left(\text { by substituting } y=g x \text { and } g(\Omega)=\Omega, g^{T} g=I\right) \\
= & \int_{\Omega} \sum_{|\alpha| \leq k}\left(D^{\alpha} u(y)\right)^{T}\left(g^{T}\right)^{\alpha}(g)^{\alpha}\left(D^{\alpha} v(y)\right)|g| d y=\langle u, v\rangle .
\end{aligned}
$$

Example 2.4 Let $\Omega \subset R^{n}$ be a bounded open domain. Assume that $\Omega$ is symmetric about the reflections with respect to the first $n-1$ axes. Let $H=W^{k, 2}(\Omega)$. Define $g: H \rightarrow H$ by

$$
(g u)\left(x_{1}, \ldots, x_{n-1}, x_{n}\right)=-u\left(-x_{1}, \ldots,-x_{n-1}, x_{n}\right) .
$$

Then $G=\{i d, g\} \cong \mathbb{Z}_{2}$.

Example 2.5 Let $\Omega \subset R^{2}$ be a bounded open domain. Let $m>1$ be an integer. For each point $x=(r, \theta) \in \Omega$ denote

$$
g x \equiv g(r, \theta)=\left(r, \theta+\frac{2 \pi}{m}\right) \quad \text { and } \quad \bar{h} x \equiv \bar{h}(r, \theta)=(r,-\theta)
$$

that is for $\alpha=\frac{2 \pi}{m}$

$$
g=\left[\begin{array}{cc}
\cos (\alpha) & -\sin (\alpha) \\
\sin (\alpha) & \cos (\alpha)
\end{array}\right] \text { and } \bar{h}=\left[\begin{array}{cc}
1 & 0 \\
0 & -1
\end{array}\right]
$$


Assume that $g(\Omega)=\Omega$ and $\bar{h}(\Omega)=\Omega$. Let $H=W^{1,2}(\Omega)$ be the Sobolev space of functions on $\Omega$ with the inner product $\langle u, v\rangle=\int_{\Omega}(\nabla u \cdot \nabla v+u v) d x$. Let $\mathbf{e o}= \pm 1$ be fixed. For each $u \in H$, we define

$$
g(u)(x)=u(g x) \quad \text { and } \quad h(u)(x)=\mathbf{e o}(\bar{h} x) .
$$

It is clear that $g$ represents a rotation and $h$ represents an even $(\mathbf{e o}=1)$ or an odd $(\mathbf{e o}=-1)$ reflection. The inner product is invariant under both linear operators $g$ and $h$. To see this let $f$ denote the operator $g$ or $h$ and the matrix $g$ or $\bar{h}$. We have $f^{T} f=I$, thus

$$
\begin{aligned}
\langle f u, f v\rangle= & \left.\int_{\Omega}(\nabla u(f x))^{T} f^{T} f \nabla v(f x)+u(f x) v(f x)\right) d x \\
& (\text { substituting } y=f x \text { and note } f(\Omega)=\Omega,|f|=1) \\
= & \int_{\Omega}(\nabla u(y) \nabla v(y)+u(y) v(y))|f| d y=\langle u, v\rangle .
\end{aligned}
$$

We have a finite group

$$
G=\left\{g, g^{2}, . ., g^{m}, h g, h g^{2}, . ., h g^{m}\right\}
$$

of linear isomorphisms of $H$ which has two generators $g$ and $h$. The invariant subspace $H_{G}$ of $H$ is defined by

$$
H_{G}=\left\{u \in H: g^{i} u=u \text { and } h g^{i} u=u \quad \forall i=1,2, \ldots, m\right\}
$$

For each $u \in H$, the Haar integral operator on $u$ is given by

$$
\mathcal{H} u=u_{G} \equiv \frac{1}{2 m}\left(\sum_{i=1}^{m} g^{i} u+\sum_{i=1}^{m} h g^{i} u\right) \in H_{G} \quad \text { and } \quad u_{G}^{\perp} \equiv u-u_{G} \in H_{G}^{\perp} .
$$

There are symmetries that cannot be defined by a compact group of linear isomorphisms of $H$, such as composite symmetries involving partially defined symmetries. We may identify those symmetries by using several projections, among which the first one is orthogonal with which computational error is expected to be minimized.

Example 2.6 Let $\Omega=[-a, a] \times[-a, a]$ in $R^{2}$ and $H=H_{0}^{1}(\Omega)$. We are interested in finding $a$ critical point $u^{*}$ with the following symmetries. The profile of $u^{*}$ is even symmetric about the line $y=-x$, even symmetric about the $x$-axis for points $(x, y)$ with $0 \geq y \geq-x \geq-a$ and even symmetric about the $y$-axis for points $(x, y)$ with $0 \geq-x \geq y \geq-a$. To define the invariant space, we combine two projection operators $T=T_{2} \cdot T_{1}$, where

$$
\left(T_{1} u\right)(x, y)=\frac{1}{2}(u(x, y)+u(-y,-x)), \quad(x, y) \in \Omega
$$


is an orthogonal projection from $H$ to $H_{T_{1}}$ with which computational error is minimized and

$$
\left(T_{2} u\right)(x, y)= \begin{cases}u(x,-y), & 0 \geq y \geq-x \\ u(-x, y), & 0 \geq-x \geq y \\ u(x, y), & \text { otherwise }\end{cases}
$$

which is a projection from $H_{T_{1}}$ to $H_{T}$. Note that $T_{2}$ can map $H$ to outside of $H$. However $T_{2}$ projects $H_{T_{1}}$ into $H$. Thus $T=T_{2} \cdot T_{1}$ projects $H$ into $H_{T} \subset H$.

Remark 2.1 When certain symmetries cannot be described by a compact group of linear isomorphisms such as the case in Example 2.6, it is difficult to analytically verify that if or not a point $u$ having the symmetries will imply that $\nabla J(u)$ has the same symmetries. But it can be numerically checked as follows. Let $T$ represent the projection operator onto $H_{I}$. The term $\nabla J(u)-T(\nabla J(u))$ represents the asymmetric part of $\nabla J(u)$ together with the computational error. Let $\varepsilon$ represent the order of the computational error. If $\|\nabla J(u)-T(\nabla J(u))\| \approx$ $\varepsilon\|\nabla J(u)\|$, it means that the asymmetric part is caused by the computational error, not the asymmetric part of $\nabla J(u)$. Thus $\nabla J(u)$ has the same symmetries. Otherwise $\nabla J(u)$ does not have the same symmetries.

\section{$3 \quad$ Numerical Examples}

In this section, we present several typical numerical examples to illustrate the theory and the numerical algorithm. Each of these examples has its own feature in symmetry and in the solution profile. These examples also exhibit two types of mathematical phenomena. The first phenomenon is about symmetry breaking in terms of some parameters of the problems. In general, for the problems with the full radial symmetry in $\mathbf{R}^{n}$, there is always a radial solution for all parameters. When we vary the parameters the problems may or may not have non-radial solutions. If non-radial solutions appear we say symmetry breaking occurs. We demonstrate this feature by using autonomous equations with the Dirichlet boundary condition (the LaneEmden equation) on thin annular domains as well as for the Henon equation in ball domains. For the existence of these solutions, some have been proved theoretically and others are still open (see [5] [4] for more references of theoretical studies). These non-radial solutions tend to have higher energy and possess large Morse indices. The second phenomenon is about nodal solutions (sign-changing solutions) for nonlinear Dirichlet problems. Again these solutions 
tend to have higher energy and large Morse indices. The existence of most of these solutions are still open (see [11] for more references of theoretical studies on nodal solutions). As these examples show our new numerical algorithm is very powerful in dealing with multiple saddle points with large Morse indices and is very efficient and stable in dealing with the presence of symmetries.

To find a saddle point of $J$, when $H$ is replaced by a $J$-invariant space $H_{I}$ in LMM, whether or not the algorithm is stable depends on whether or not the support $L$ in $H_{I}$ is sufficient. We will pay special attention to the case where $L=\{0\}$, the smallest possible support. So we have tried to explore the symmetries of a problem to the maximum. It is known that when $L=\{0\}$, our solution set coincides with the Nehari manifold in $H_{G}$ defined by

$$
\mathcal{M}=\left\{t_{u} u: u \in H_{G},\|u\|=1, t_{u}>0, \nabla J\left(t_{u} u\right) \perp u\right\} .
$$

Under some standard conditions, it can be shown [12] that $\mathcal{M}$ is smooth and the peak selection $p$ is unique and $C^{1}$, which gives us a great advantage to show the convergence of LMM [13].

Let $\Omega \subset R^{2}$ be a bounded open domain. Consider the Lane-Emden equation

$$
\begin{cases}\Delta u(x)+u^{p}(x)=0, & x \in \Omega, \\ u(x)=0, & x \in \partial \Omega,\end{cases}
$$

with $p=3$ and the Henon equation

$$
\begin{cases}\Delta u(x)+|x|^{q} u^{p}(x)=0, & x \in \Omega, \\ u(x)=0, & x \in \partial \Omega,\end{cases}
$$

with $p=3$ and $q>0$. Those two equations have quite different features due to whether or not depending on $x$ explicitly. The solutions to (3.1) prefer open space, while the solutions to (3.2) seem to prefer corners.

Though the abstract theory asserts the invariance under general conditions of the group action, we demonstrate for the examples above how to verify that for certain symmetries, if $u$ has a symmetry, then $\nabla J(u)$ possesses the same symmetry. Let $G \subset \mathcal{O}(n)$ be a closed subgroup and $\Omega$ is invariant under the action of $G$. Let $\lambda_{1}<\lambda_{2}<\lambda_{3}<\ldots$ be the eigenvalues of $-\Delta$ with Dirichlet boundary condition and $E_{i}$ be the eigenspace corresponding to $\lambda_{i}$. Then for $u \in E_{i},-\Delta u=\lambda_{i} u$. It follows from this that for $g \in G$ we also have $-\Delta g u=\lambda_{i} g u$. That is, $E_{i}$ is invariant subspace under $G$. This means $H_{I}$ and $H_{I}^{\perp}$ are both generated by invariant eigenspaces. Now let $f(x, u)=|x|^{q} u^{p}$. Then it is easy to verify that

$$
(\nabla J(u), v)=\int_{\Omega} \nabla\left(u-(-\Delta)^{-1} f(x, u)\right) \nabla v d x
$$


which implies $\nabla J(u)=u-(-\Delta)^{-1} f(x, u)$. Assume $u \in H_{I}$ is in the invariant space of the action $G$. Then $f(x, u) \in H_{I}$ and we have $\nabla J(u) \in H_{I}$ if and only if $w=(-\Delta)^{-1} f(x, u) \in H_{I}$. Let $w=w_{I}+w_{I}^{\perp}$ with $w_{I} \in H_{I}$ and $w_{I}^{\perp} \in H_{I}^{\perp}$. Since $w$ solves for $-\Delta w=f(x, u) \in H_{I}$ we have $-\Delta w_{I}^{\perp}=0$, or $w_{I}^{\perp}=0$ and then $w \in H_{I}$. This gives $\nabla J(u) \in H_{I}$.

In the examples we shall consider several types of domains:

(a) $\Omega=[-1,1] \times[-1,1]$, a squarer;

(b) $\Omega=[-1.5,1.5] \times[-1,1]$, a rectangle;

(c) $\Omega=\left\{(x, y) \in R^{2}: x^{2}+y^{2} \leq 1\right\}$, the unit disk and

(d) $\Omega=\left\{(x, y) \in R^{2}: 0.7 \leq \sqrt{x^{2}+y^{2}} \leq 1\right\}$, an annulus.

The first two types of domains are used to exploit the structure of nodal solutions which are saddle points of the functional with large Morse indices. The latter two types of domains are discs and annular domains which will be used to demonstrate symmetry breaking phenomena and to find non-radial positive solutions while radial positive solutions always exist. Disks and annular domains are the most symmetric domains in $R^{2}$, i.e., they are invariant under the full $\mathcal{O}(2)$ symmetry. But the symmetry causes degeneracy, due to the fact that a rotation of a non-radial solution about any angle is still a solution. Thus the solutions are not isolated in the whole space $H$. When symmetry is properly used to define an invariant space $H_{I}$, a solution in $H_{I}$ can be isolated.

It is known that on a disk, the Lane-Emden equation has a unique positive solution as the ground state which is radially symmetric and has a single peak; while the Henon equation, in addition to the radially symmetric positive solution which has the highest critical level among all other positive solutions if exist, may have a non-radially symmetric positive solution. The existence of a non-radial solution to the Henon equation depends on the parameter $q$, which is a typical symmetry breaking phenomenon. There may have multi-peak positive solutions. The number of peaks that a positive solution may have depends on the parameter $q$. This may be considered a bifurcation problem with parameter $q$. Due to high critical level, the radially symmetric positive solution is the most unstable and therefore most elusive to capture among all positive solutions by LMM, since it will never get a sufficient support, unless one uses the radial symmetry to convert it into solving an ordinary differential equation. However, the method developed in this paper can easily capture this radially symmetric solution. 
We point out that for a numerical computation using symmetry to be successful, it is important that its discretized mesh points must match the symmetry. In the numerical examples, $\varepsilon<10^{-4}$ is used to terminate iterations and an initial guess $v_{0}^{1}$ is obtained from solving

$$
\Delta v(x)=c(x), x \in \Omega \quad \text { and } \quad v(x)=0, x \in \partial \Omega
$$

where $c(x)$ is equal to $-1(+1)$ if one wants $v(x)$ to be convex down (up) at $x$ and is equal to 0 if the profile of $v(x)$ is not of concern at $x$. In all the figures, $J$ is the critical value. Figs.1-9 are solutions to the Lane-Emden equation and we mainly want to demonstrate a variety of nodal solutions which are saddle points having large Morse indices. Figs.10-11 are positive solutions of the Lane-Emden equation on annular domains. Figs.12-27 are solutions to the Henon equation, for these we explore the symmetry breaking phenomenon here by showing that as the parameter $q$ increases more and more non-radial positive solutions appear which should also have large Morse indices. The following is a list of examples for which we used symmetries to find each of the solutions.

(1) cf. Fig. 1. No symmetry is needed or $(\mathcal{H} u)(x, y)=u(x, y) . \quad L=\{0\}$. This is a Mountain-Pass solution and its Morse index is one.

(2) cf. Fig. 3. Either odd reflection about the line $y=x$ or odd reflection about the origin. $(\mathcal{H} u)(x, y)=\frac{1}{2}(u(x, y)-u(-y,-x))$ or $(\mathcal{H} u)(x, y)=\frac{1}{2}(u(x, y)-u(-x,-y)) . L=\{0\}$. This is a solution whose Morse index is at least two in the full space, and its Morse index is one when restricted in the invariant space.

(3) cf. Fig. 2. Odd reflection about the x-axis. $(\mathcal{H} u)(x, y)=\frac{1}{2}(u(x, y)-u(x,-y)) . L=\{0\}$.

(4) cf. Fig. 4. Either odd reflections about the $x$-axis and the $y$-axis, or odd symmetry about the rotation by $\frac{\pi}{2}$. $(\mathcal{H} u)(x, y)=\frac{1}{4}(u(x, y)-u(-x, y)+u(-x,-y)-u(x,-y))$ or $(\mathcal{H} u)(\theta, r)=\frac{1}{4}\left(u(\theta, r)-u\left(\theta+\frac{\pi}{2}, r\right)+u(\theta+\pi, r)-u\left(\theta+\frac{3 \pi}{2}, r\right)\right) . L=\{0\}$. This solution has Morse index at least 4 in the full space though the Morse index in the invariant space is one.

(5) cf. Fig. 5. Odd reflections about the lines $\mathrm{y}=\mathrm{x}$ and $\mathrm{y}=-\mathrm{x} . \quad L=\{0\}$ $(\mathcal{H} u)(x, y)=\frac{1}{4}(u(x, y)-u(y, x)+u(-x,-y)-u(-y,-x))$.

(6) cf. Fig. 6. Either even reflections about the x-axis and the y-axis, or even symmetry about the rotation by $\frac{\pi}{2} . L=\left\{u_{1}\right\}$ and $u_{1}$ is in Fig. 1 . 
$(\mathcal{H} u)(x, y)=\frac{1}{4}(u(x, y)+u(-x, y)+u(-x,-y)+u(x,-y))$ or

$(\mathcal{H} u)(\theta, r)=\frac{1}{4}\left(u(\theta, r)+u\left(\theta+\frac{\pi}{2}, r\right)+u(\theta+\pi, r)+u\left(\theta+\frac{3 \pi}{2}, r\right)\right)$. This is an example for which the Morse index in the invariant space is at least two.

(7) cf. Fig. 7. Odd reflections about the $\mathrm{x}$-axis, the $\mathrm{y}$-axis, the lines $\mathrm{y}=\mathrm{x}$ and $\mathrm{y}=-\mathrm{x}$. $\mathcal{H}=\mathcal{H}_{4} \mathcal{H}_{3} \mathcal{H}_{2} \mathcal{H}_{1}$ where $\left(\mathcal{H}_{1} u\right)(x, y)=\frac{1}{2}(u(x, y)-u(x,-y)),\left(\mathcal{H}_{2} u\right)(x, y)=\frac{1}{2}(u(x, y)-$ $u(-x, y)),\left(\mathcal{H}_{3} u\right)(x, y)=\frac{1}{2}(u(x, y)-u(y, x)),\left(\mathcal{H}_{4} u\right)(x, y)=\frac{1}{2}(u(x, y)-u(-y,-x))$. $L=\{0\}$.

(8) cf. Fig. 8. Odd reflections about the x-axis and the y-axis. $(\mathcal{H} u)(x, y)=\frac{1}{4}(u(x, y)-$ $u(-x, y)+u(-x,-y)-u(x,-y)) . L=\{0\}$. Since the domain is a rectangle, the rotation by $\frac{\pi}{2}$ is not applicable. Contrast to Fig. 4.

(9) cf. Fig. 9. Even reflections about the x-axis and the y-axis. $(\mathcal{H} u)(x, y)=\frac{1}{4}(u(x, y)+$ $u(-x, y)+u(-x,-y)+u(x,-y)) . L=\left\{u_{1}\right\}$ where $u_{1}$ is the single peak positive solution in the rectangle. This is the same symmetry used in example Fig. 6. It is interesting to compare to Fig. 6. If we let the rectangle $[-1.5,1.5] \times[-1,1]$ change gradually to the square $[-1,1] \times[-1,1]$, e.g., $[-1.01,1.01] \times[-1,1]$, the solution remains of the same profile; It is an interesting observation. If we compare the critical values, profiles and symmetries of Figs. 4 and 6 with that of Figs. 8 and 9, we note that their sequential orders in critical level and profiles have changed drastically due to even a slight change in the geometry of the domain.

(10) cf. Fig. 10. Rotation symmetry by $\frac{2 \pi}{3} \cdot(\mathcal{H} u)(\theta, r)=\frac{1}{3}\left(u(\theta, r)+u\left(\theta+\frac{2 \pi}{3}, r\right)+u\left(\theta+\frac{4 \pi}{3}, r\right)\right)$. $L=\{0\}$. Such a solution failed to be captured in [12] due to the fact that without using the symmetry, a sufficient support $L$ in $H$ contains infinitely many saddle points at lower critical level. We observe that the solution generated not only has the $Z_{3}$ symmetry it also has the additional symmetry of being even about the rotation by $\frac{2 \pi}{3}$. Thus the solution has $D_{3}$ symmetry as well. With our new algorithm we may capture, in a stable way, solutions with $D_{k}$ symmetry for any prime number $k$. The Morse indices of these solutions should be large depending upon the number of peaks $k$. This is related to the symmetry breaking phenomena for radially symmetric elliptic problems. The existence and qualitative behavior of these solutions can be found in [5] and references therein. A rotation of the solution by any angle is still a solution. It is a degenerate case. However, adding an even symmetry about the $\mathrm{x}$-axis can bypass the degeneracy. 
(11) cf. Fig. 11. Same symmetry as in Example 2.6. The existence of such solution is still open. Following Remark 2.1, it has been numerically checked that for each $v^{k}=p\left(u^{k}\right) \in$ $H_{I}$ generated by LMM, we have $\nabla J\left(v^{k}\right) \in H_{I}$, i.e., the invariant subspace $H_{I}$ is welldefined. However, this problem is degenerate and also ill-conditioned in the sense that if we fix one peak and move another peak around, the change in the function value $J$ is almost invisible.

(12) cf. Fig. 12. No symmetry is needed. $L=\{0\}$. This radial solution should be the unique positive solution to (3.2). A non-radial solution can be found for $q \geq 1$.

(13) cf. Fig. 13. No symmetry is needed. $L=\{0\}$. However adding an even symmetry about the $\mathrm{x}$-axis will bypass the degeneracy. This is the least energy solution of the problem and this exhibits the phenomenon of symmetry breaking of ground state solutions $([4])$.

(14) cf. Fig. 14. Even symmetry about the origin or rotation by $\pi \cdot(\mathcal{H} u)(x, y)=\frac{1}{2}(u(x, y)+$ $u(-x,-y)) . L=\{0\}$. Such a radially symmetric solution is impossible to capture without using the symmetry since it has the highest critical value among all positive solutions and a sufficient support $L$ in $H$ needs to contain infinitely many positive solutions. A traditional way to find this solution is to use the radial symmetry to convert it into an ODE. On the other hand, this also shows that for small $q$ (in this case $q=3$ ) the radial solution is still the least energy solution in the class of even functions, and when we increase $q$ to $q=4$ as in the next example, the radial solution is going to lose its stability and the least energy solution becomes non-radial again.

(15) cf. Fig. 15. Either even symmetry about the origin or even symmetry about the rotation by $\pi .(\mathcal{H} u)(x, y)=\frac{1}{2}(u(x, y)+u(-x,-y)) . L=\{0\}$. Adding an even symmetry about the $\mathrm{x}$-axis or $\mathrm{y}$-axis will bypass the degeneracy. A symmetry about the rotation by $\frac{2 \pi}{3}$ with $L=\{0\}$ will generate the radially symmetric solution. From this example on, the solutions demonstrated should have large Morse indices depending upon the number of peaks of the solutions.

(16) cf. Fig. 16. Symmetry about the rotation by $\frac{2 \pi}{3}$ with $L=\{0\} \cdot(\mathcal{H} u)(\theta, r)=\frac{1}{3}(u(\theta, r)+$ $\left.u\left(\theta+\frac{2 \pi}{3}, r\right)+u\left(\theta+\frac{4 \pi}{3}, r\right)\right)$. Adding an even symmetry about the $\mathrm{x}$-axis will bypass the degeneracy. A symmetry about the rotation by $\frac{\pi}{2}$ with $L=\{0\}$ will generate the radially symmetric solution. This implies the radial solution is the ground state in the class of 
functions invariant under $Z_{4}$, but is not the ground state in the class of $Z_{3}$-invariant functions.

(17) cf. Fig. 17. Symmetry about the rotation by $\frac{\pi}{2}$ with $L=\{0\} \cdot(\mathcal{H} u)(\theta, r)=\frac{1}{4}(u(\theta, r)+$ $\left.u\left(\theta+\frac{\pi}{4}, r\right)+u\left(\theta+\frac{\pi}{2}, r\right)+u\left(\theta+\frac{3 \pi}{4}, r\right)\right)$. Adding an even symmetry about the $\mathrm{x}$-axis or $\mathrm{y}$-axis will bypass the degeneracy.

(18) cf. Fig. 18. No symmetry is needed or $\mathcal{H} u(x, y)=u(x, y) . L=\{0\}$.

(19) cf. Fig. 19. Even reflection about the y-axis. $(\mathcal{H} u)(x, y)=\frac{1}{2}(u(x, y)+u(-x, y))$. $L=\{0\}$.

(20) cf. Fig. 20. Even reflection about the line $\mathrm{y}=-\mathrm{x} .(\mathcal{H} u)(x, y)=\frac{1}{2}(u(x, y)+u(-y,-x))$. $L=\{0\}$.

(21) cf. Fig. 21. Even reflection about the x-axis and the $y$-axis. $(\mathcal{H} u)(x, y)=\frac{1}{4}(u(x, y)+$ $u(-x, y)+u(-x,-y)+u(x,-y)) . L=\{0\}$.

(22) cf. Fig. 22. Odd reflection about the y-axis. $(\mathcal{H} u)(x, y)=\frac{1}{2}(u(x, y)-u(-x, y)) . L=\{0\}$.

(23) cf. Fig. 23. Odd reflection about the line $\mathrm{y}=-\mathrm{x} .(\mathcal{H} u)(x, y)=\frac{1}{2}(u(x, y)-u(-y,-x))$. $L=\{0\}$.

(24) cf. Fig. 24. Even reflection about the y-axis and odd reflection about the x-axis. $L=\{0\}$.

(25) cf. Fig. 25. Odd reflections about the x-axis and the y-axis. $(\mathcal{H} u)(x, y)=\frac{1}{4}(u(x, y)+$ $u(-x, y)-u(-x,-y)-u(x,-y)) . L=\{0\}$.

(26) cf. Fig. 26. The same symmetry used in finding Fig. 7. $L=\{0\}$.

(27) cf. Fig. 27. The same symmetry as in Example 2.6 and also in (11). It is an interesting example, since its symmetry is partial and cannot be described by a compact group. Theoretically the existence of such a solution is still open. However we are able to follow Remark 2.1 to numerically verify the invariant subspace in the sense of Definition 2.1. For each $v^{k}=p\left(u^{k}\right) \in H_{I}$ generated in LMM, we find that the asymmetric part of $d^{k}=\nabla J\left(v^{k}\right)$ satisfies $\|$ Asymmetric part of $d^{k}\left\|_{H_{0}^{1}} \cong 2 . \cdot 10^{-4}\right\| d^{k} \|_{H_{0}^{1}}$. Thus we are very optimistic about the existence of this solution. 

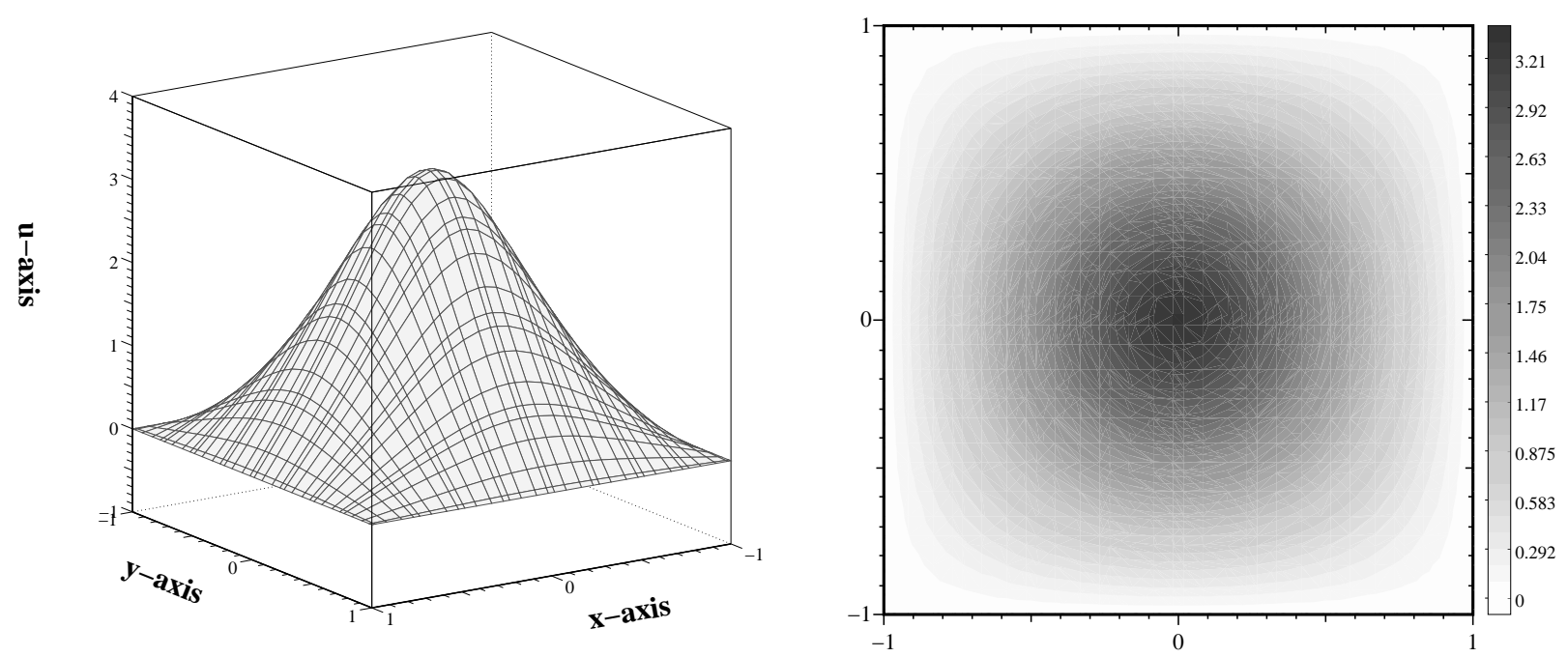

Figure 1: A solution to $(3.1), J=9.441$
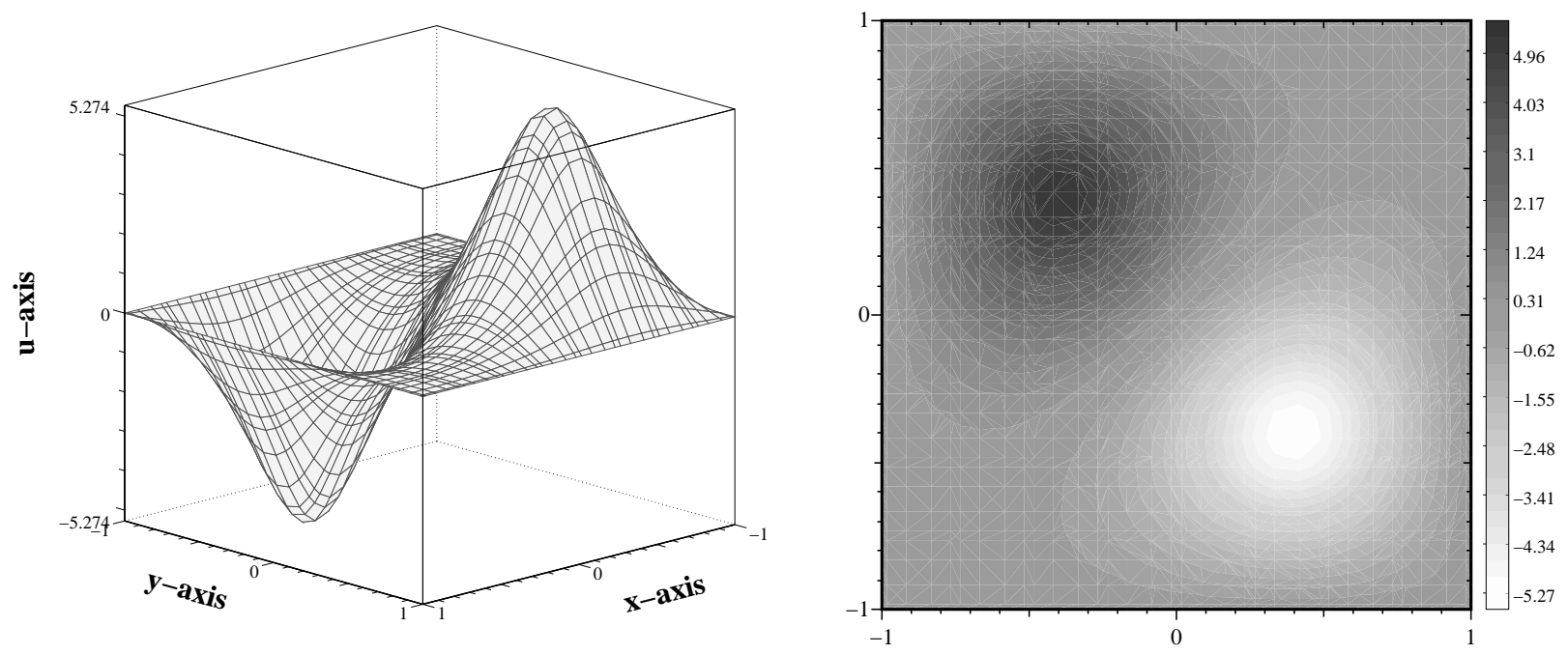

Figure 2: A solution to $(3.1), J=48.81$

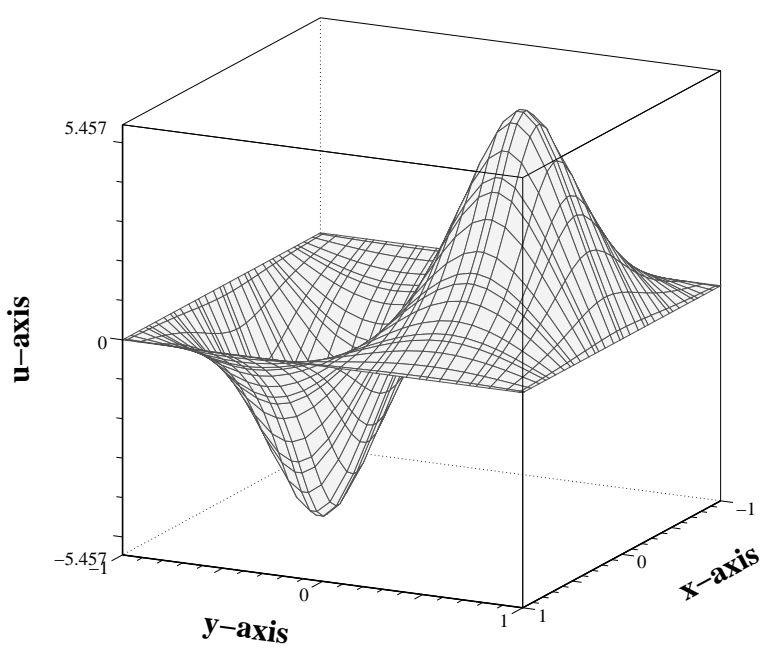

Figure 3: A solution to $(3.1), J=53.58$

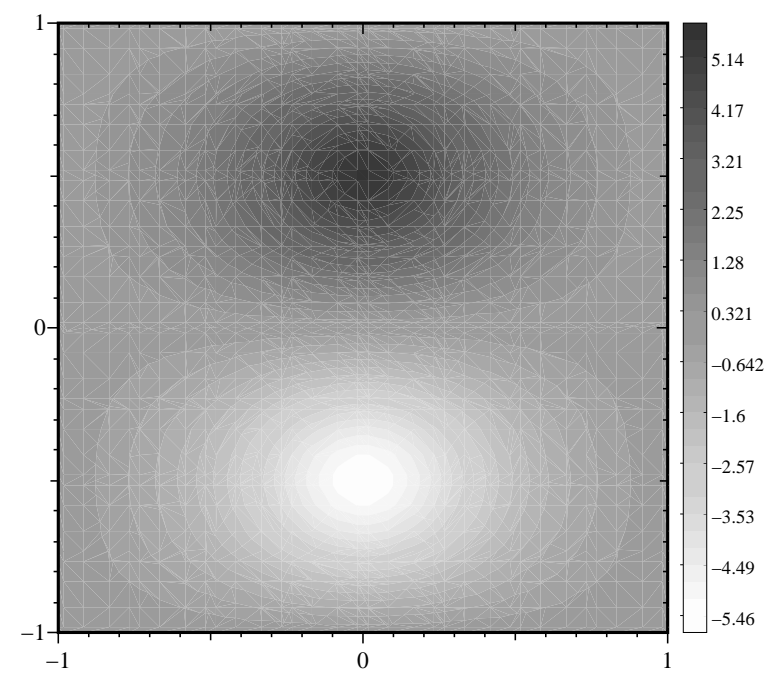



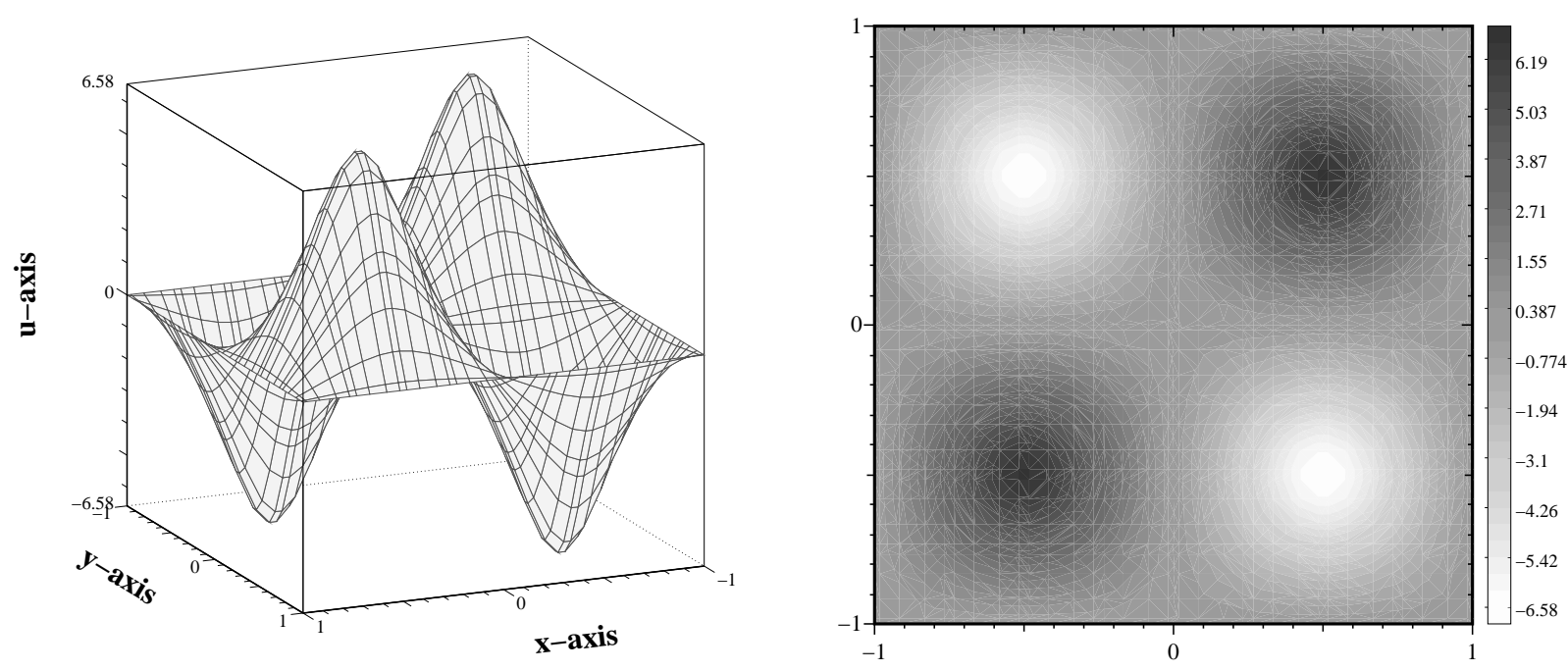

Figure 4: A solution to (3.1), $J=151.0$
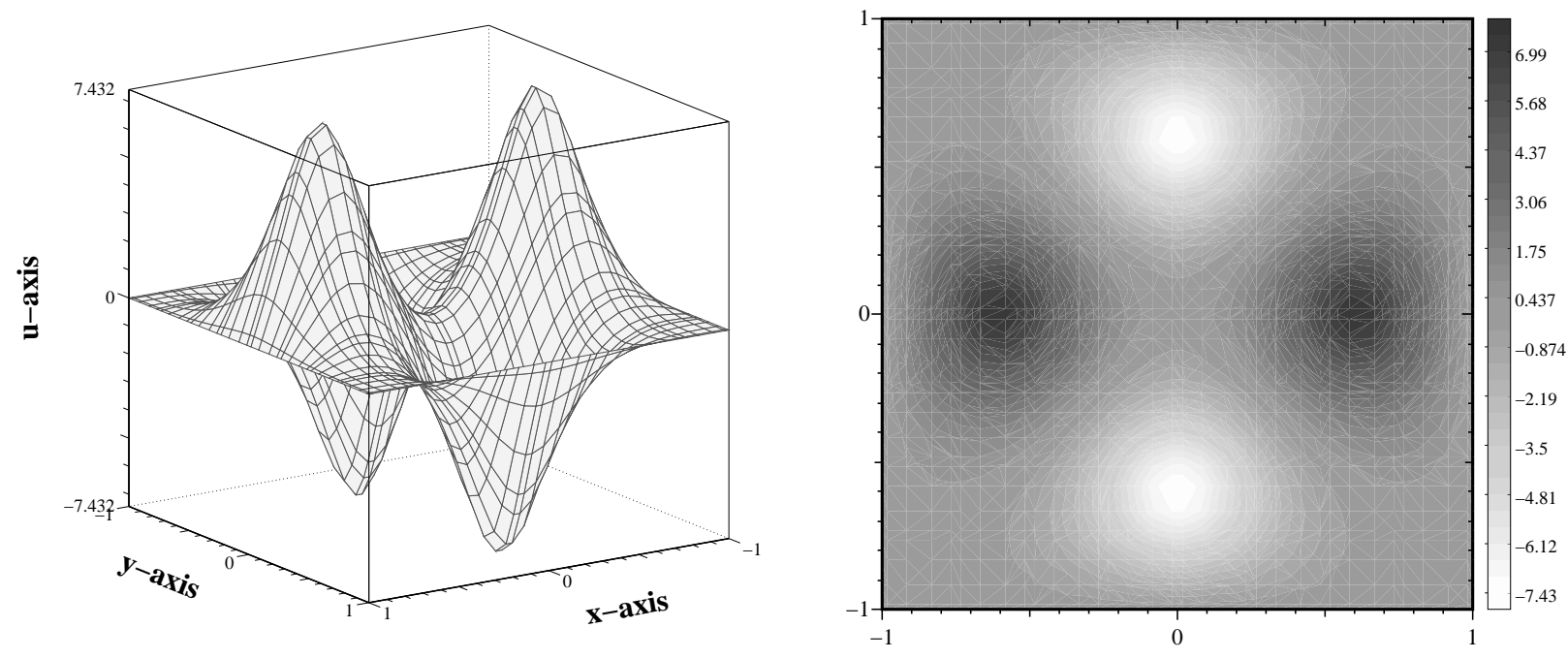

Figure 5: A solution to (3.1), $J=195.1$

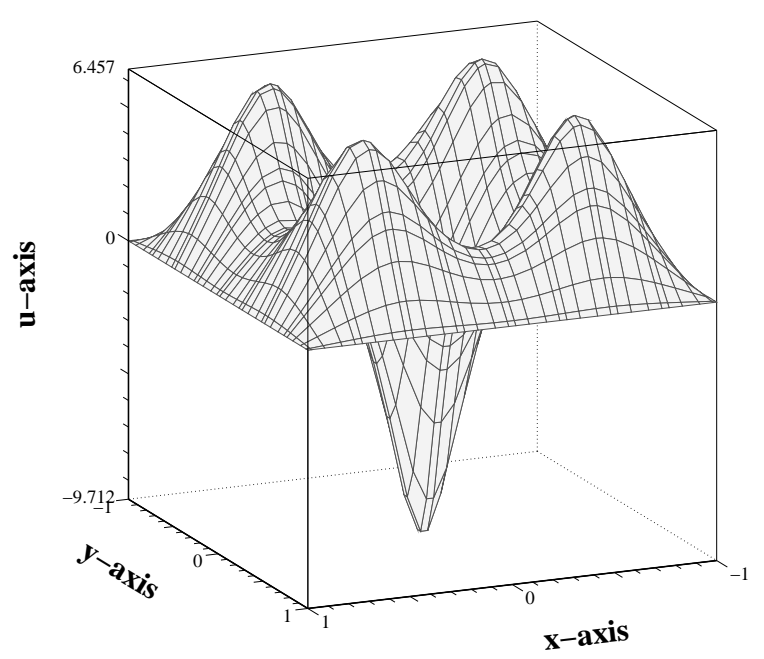

Figure 6: A solution to (3.1), $J=233.0$

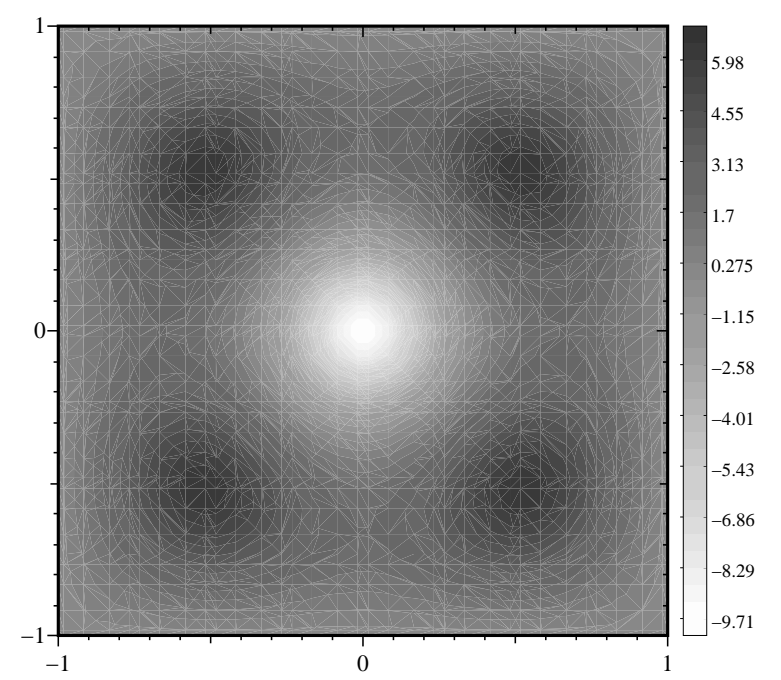



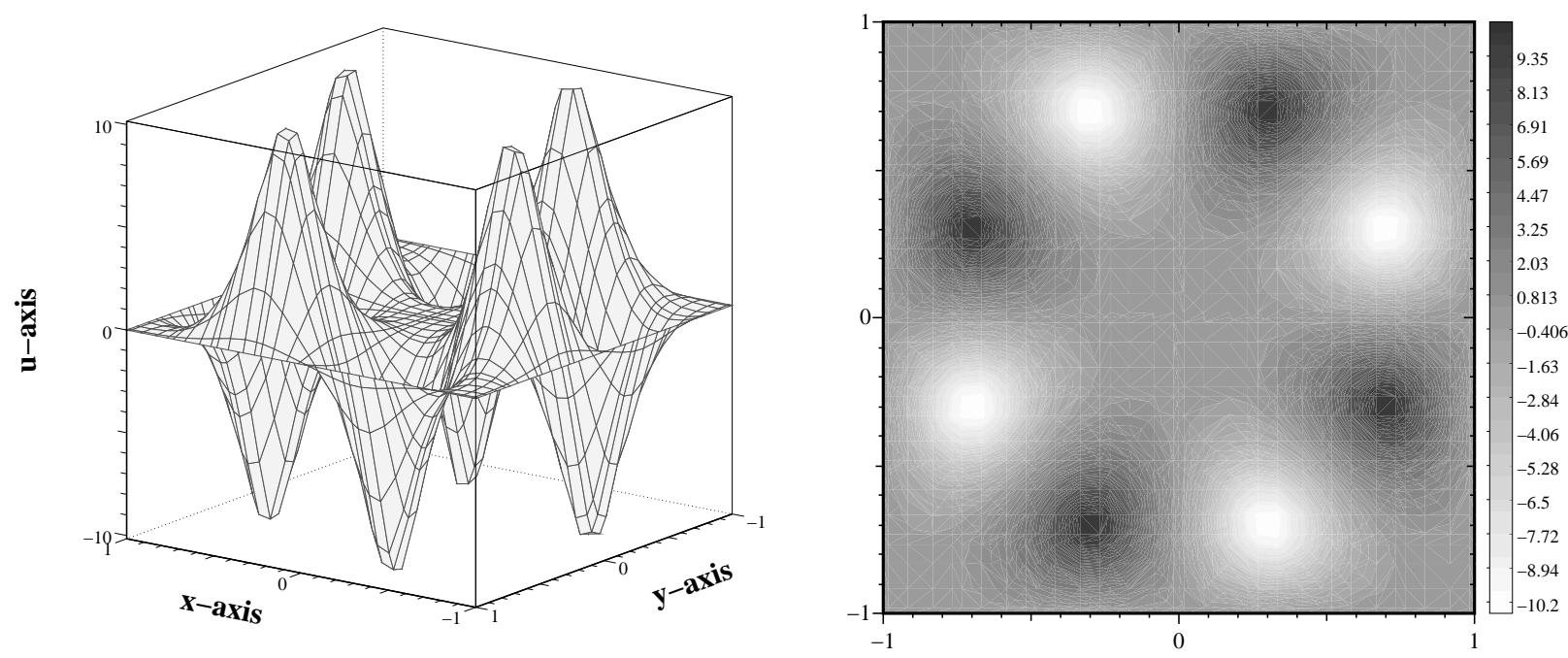

Figure 7: A solution to (3.1), $J=777.4$
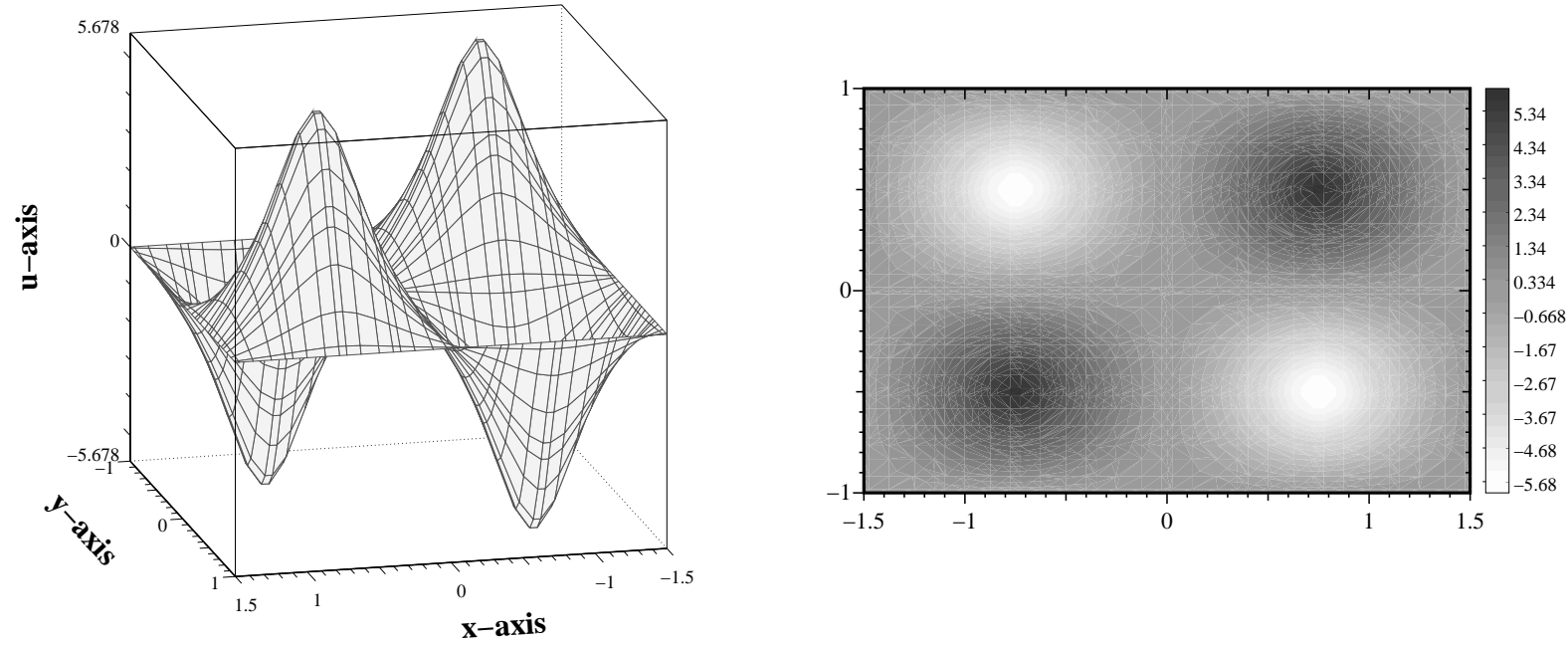

Figure 8: A solution to $(3.1), J=114.7$
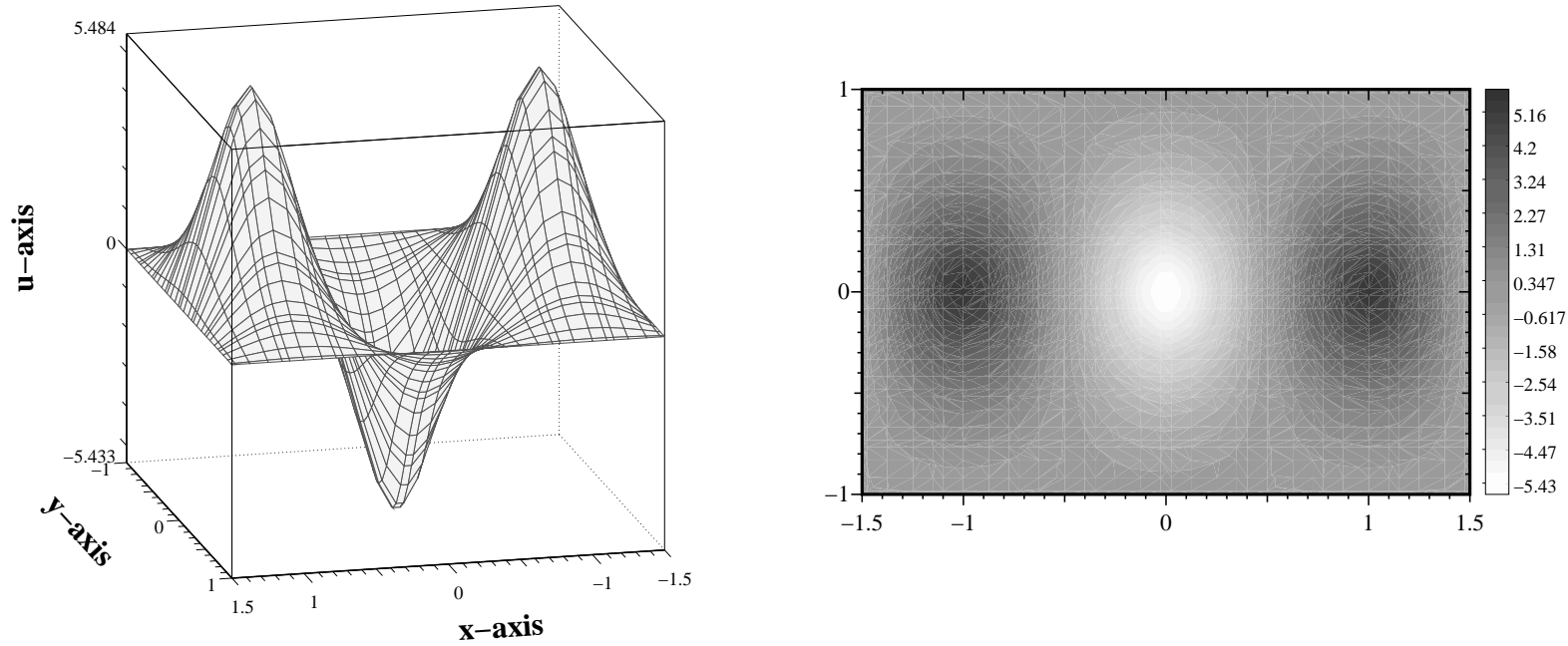

Figure 9: A solution to $(3.1), J=80.34$ 

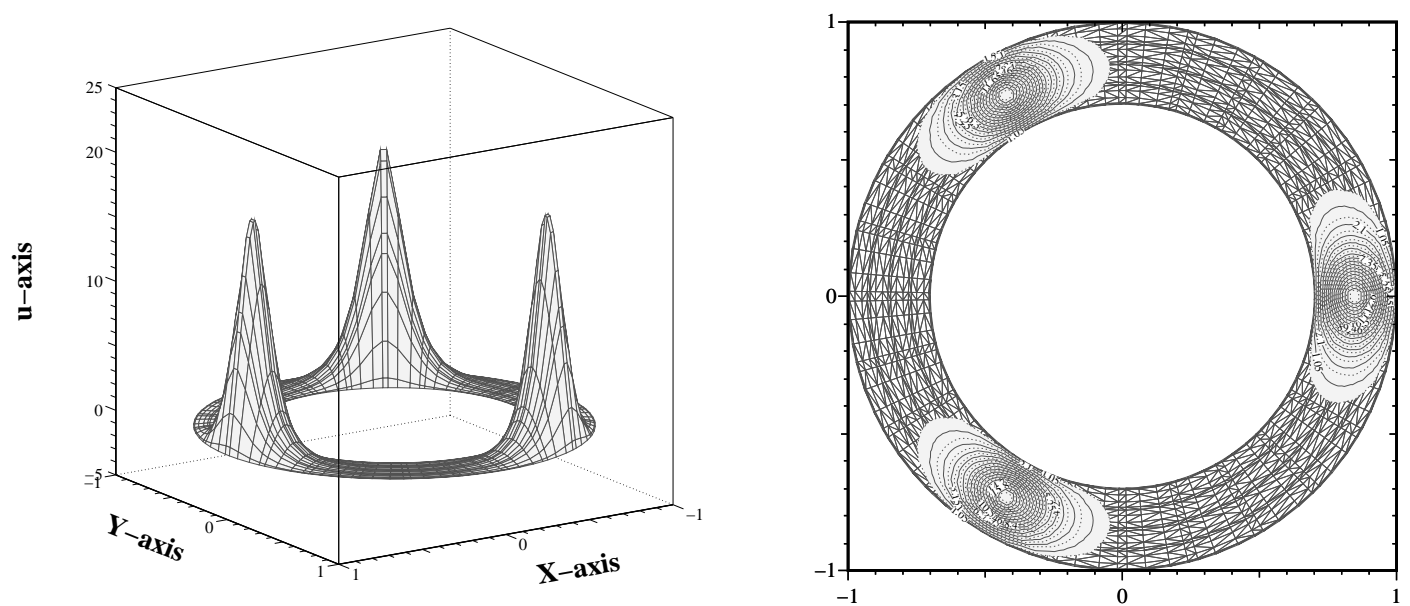

Figure 10: A solution to (3.1), $J=870.7$
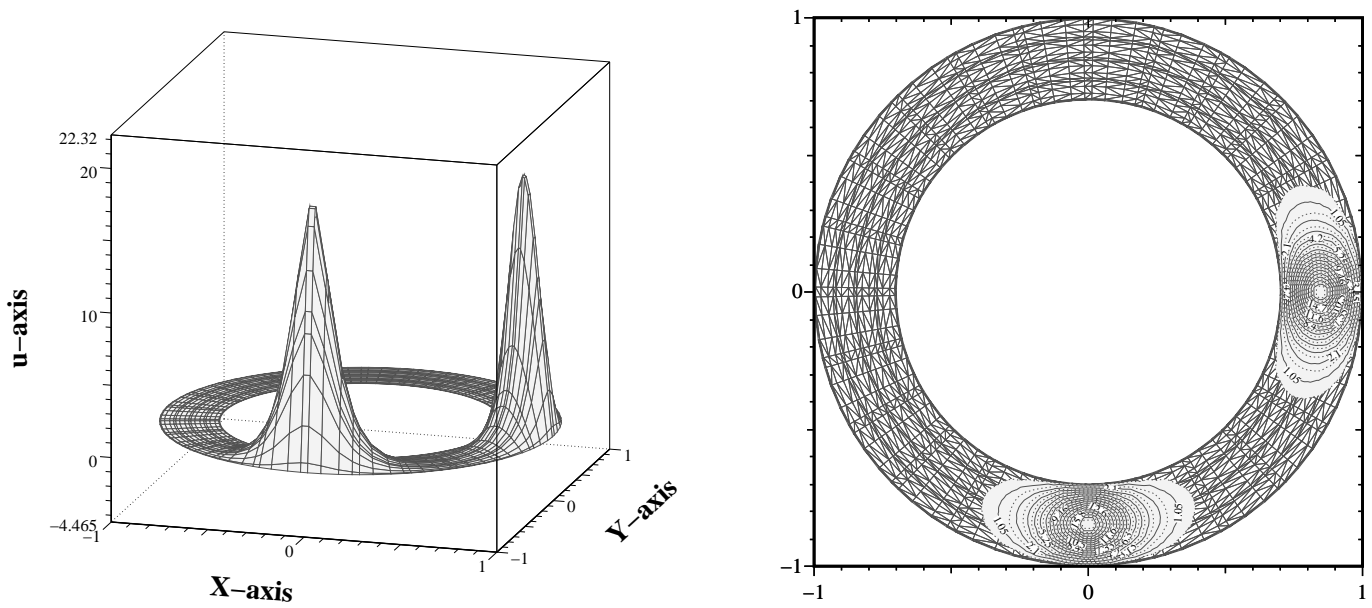

Figure 11: A solution to (3.1), $J=580.5$
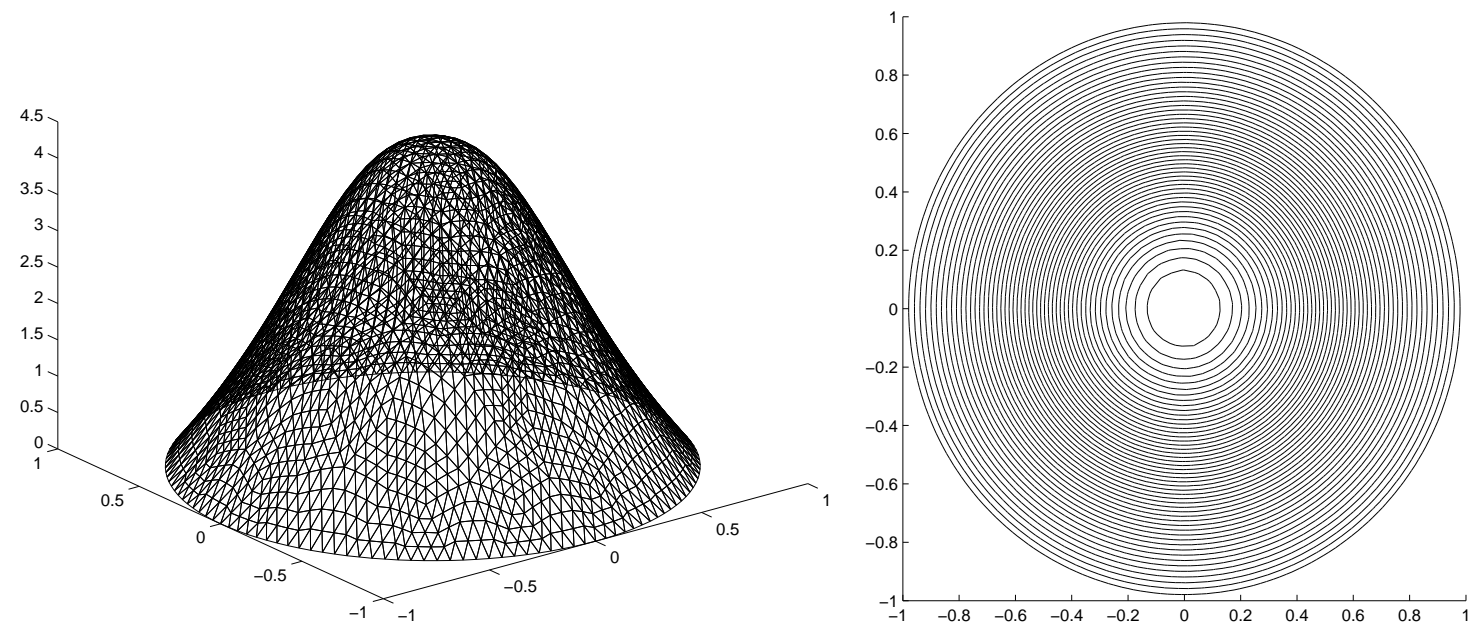

Figure 12: A solution to (3.2) with $q=0.5, J=21.5346$ 

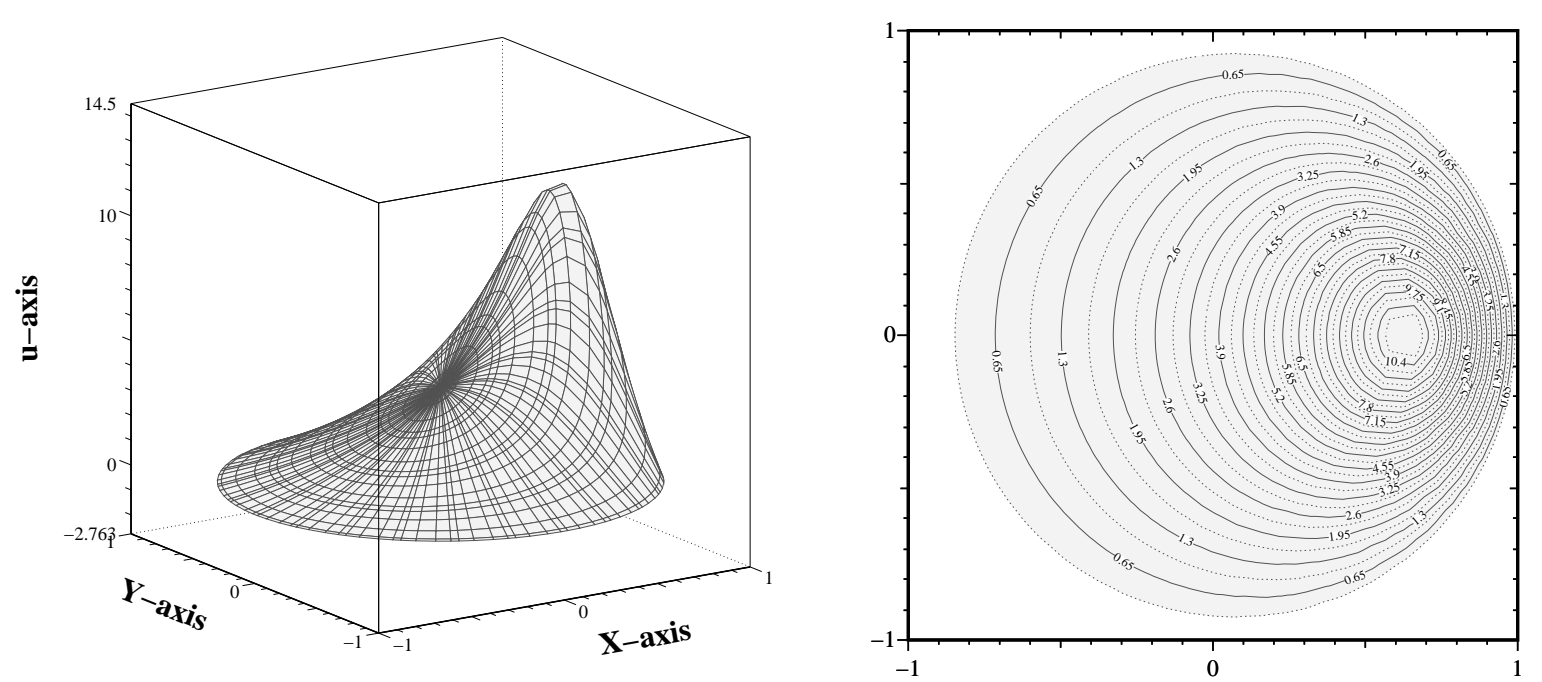

Figure 13: A solution to (3.2) with $q=3, J=114.4$
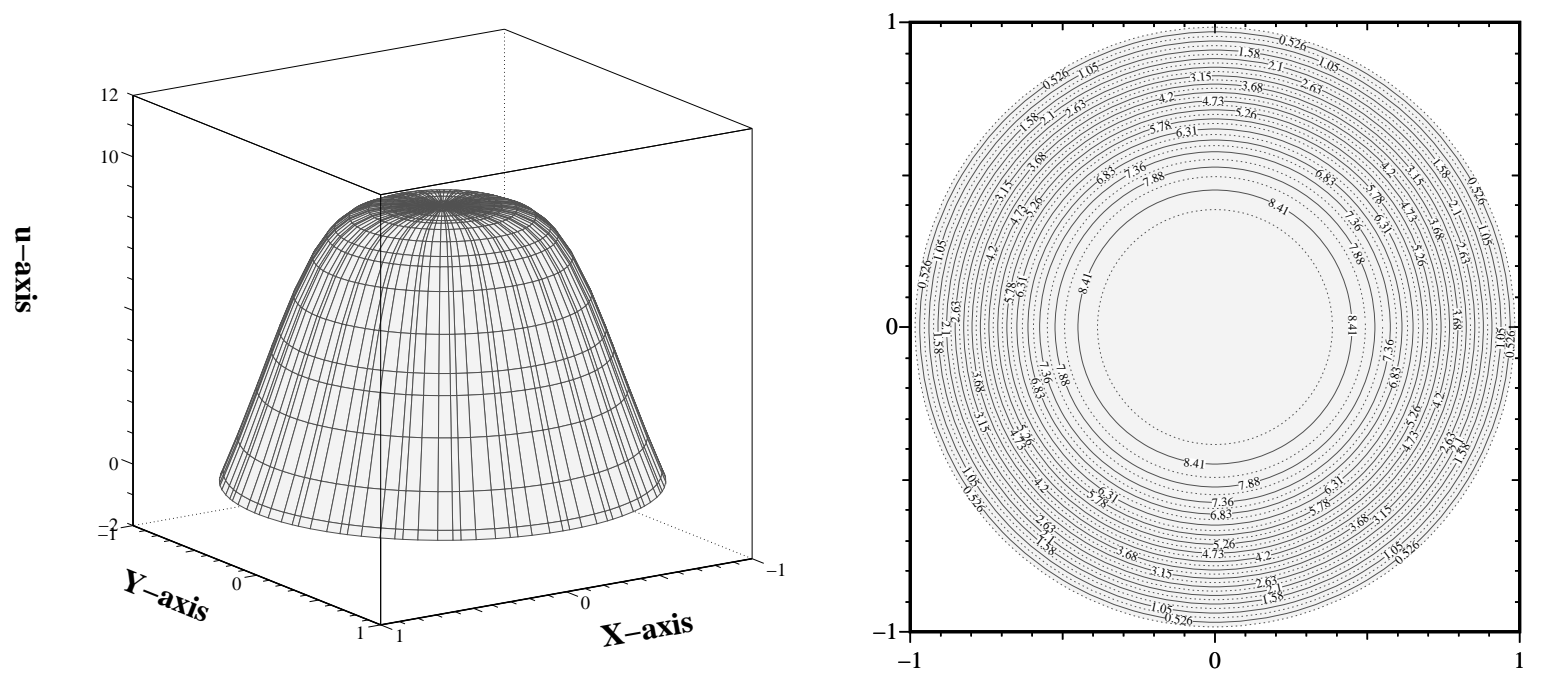

Figure 14: A solution to (3.2) with $q=3, J=171.7$
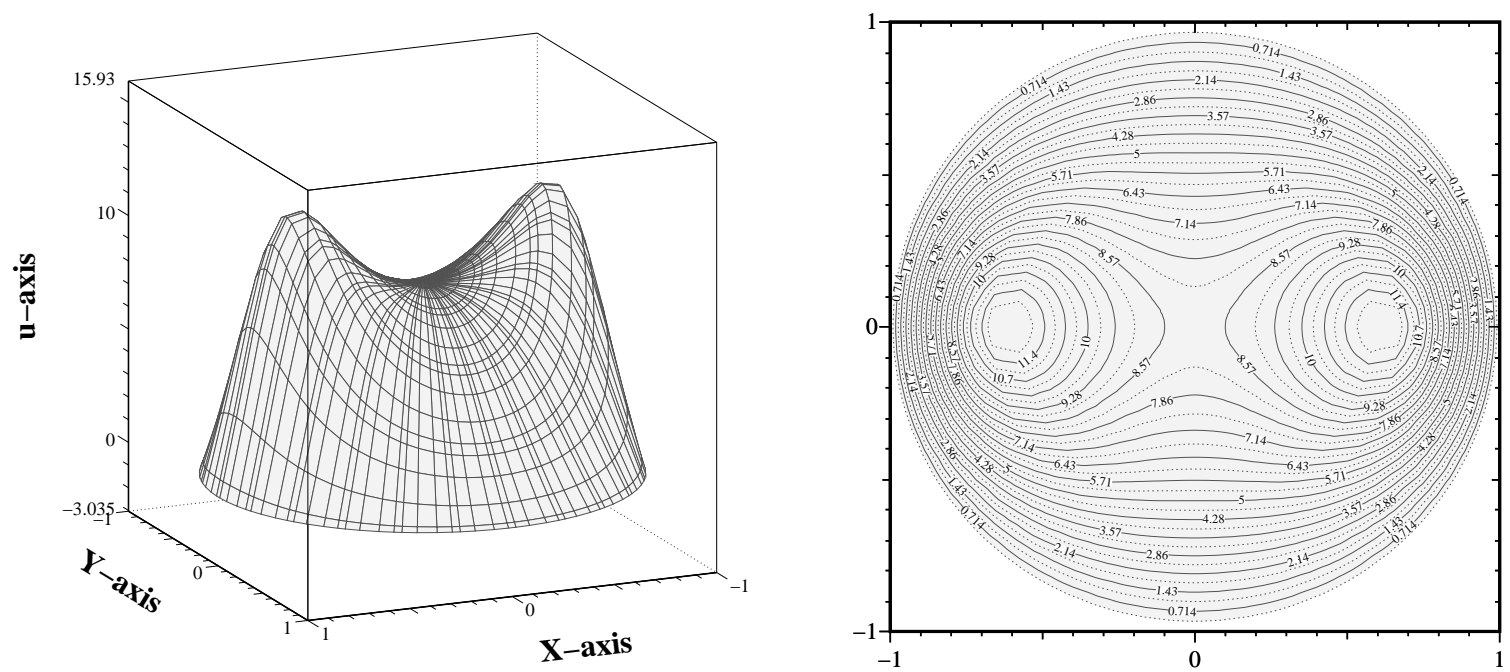

Figure 15: A solution to (3.2) with $q=4, J=285.9$ 

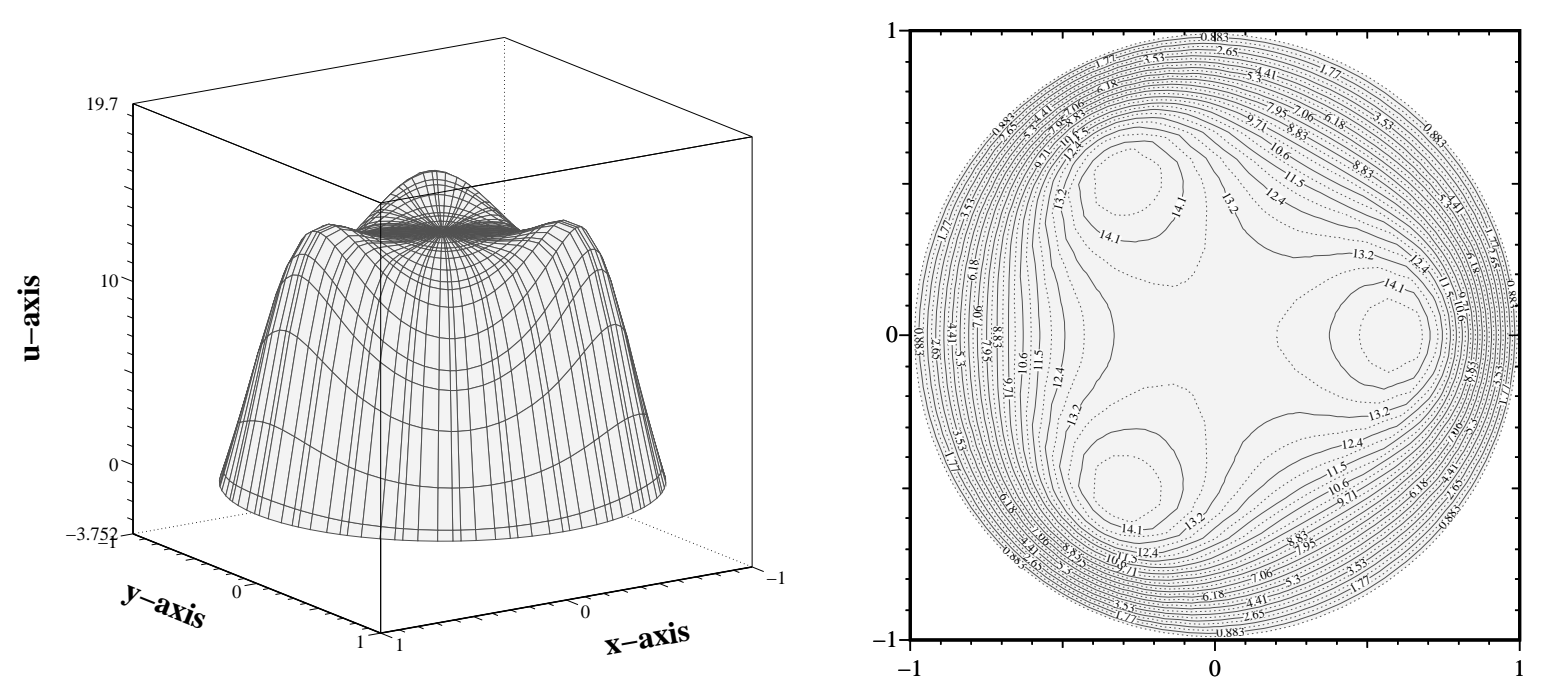

Figure 16: A solution to (3.2) with $q=6, J=702.2$
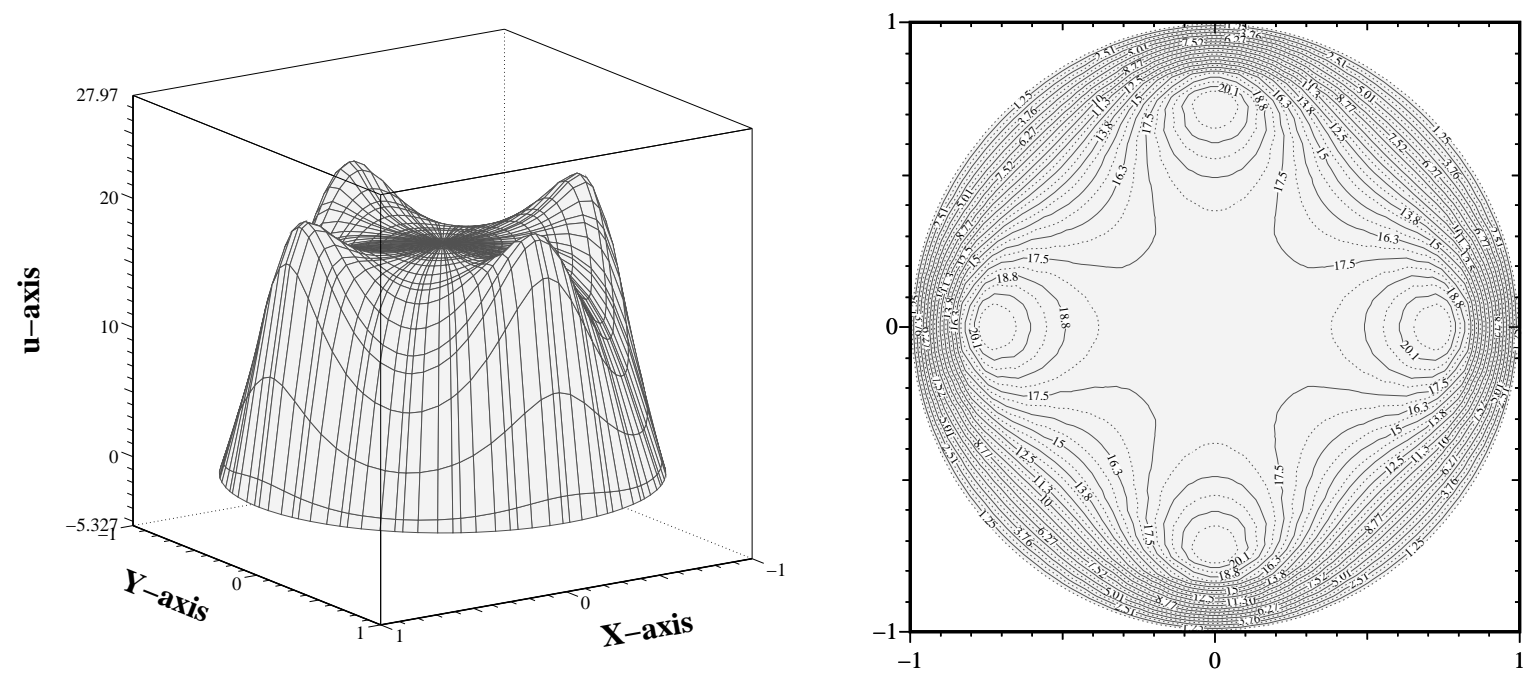

Figure 17: A solution to (3.2) with $q=9, J=1815$.
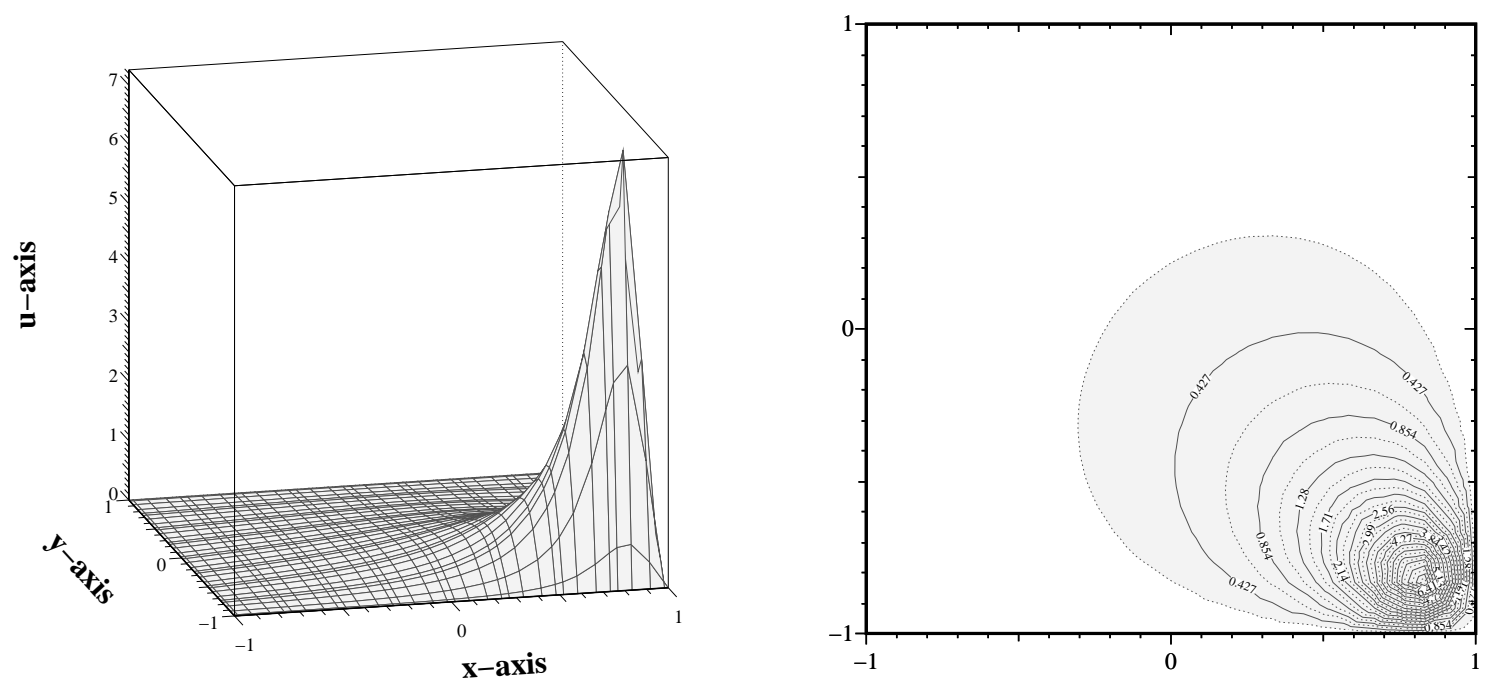

Figure 18: A solution to (3.2) with $q=9, J=43.44$ 

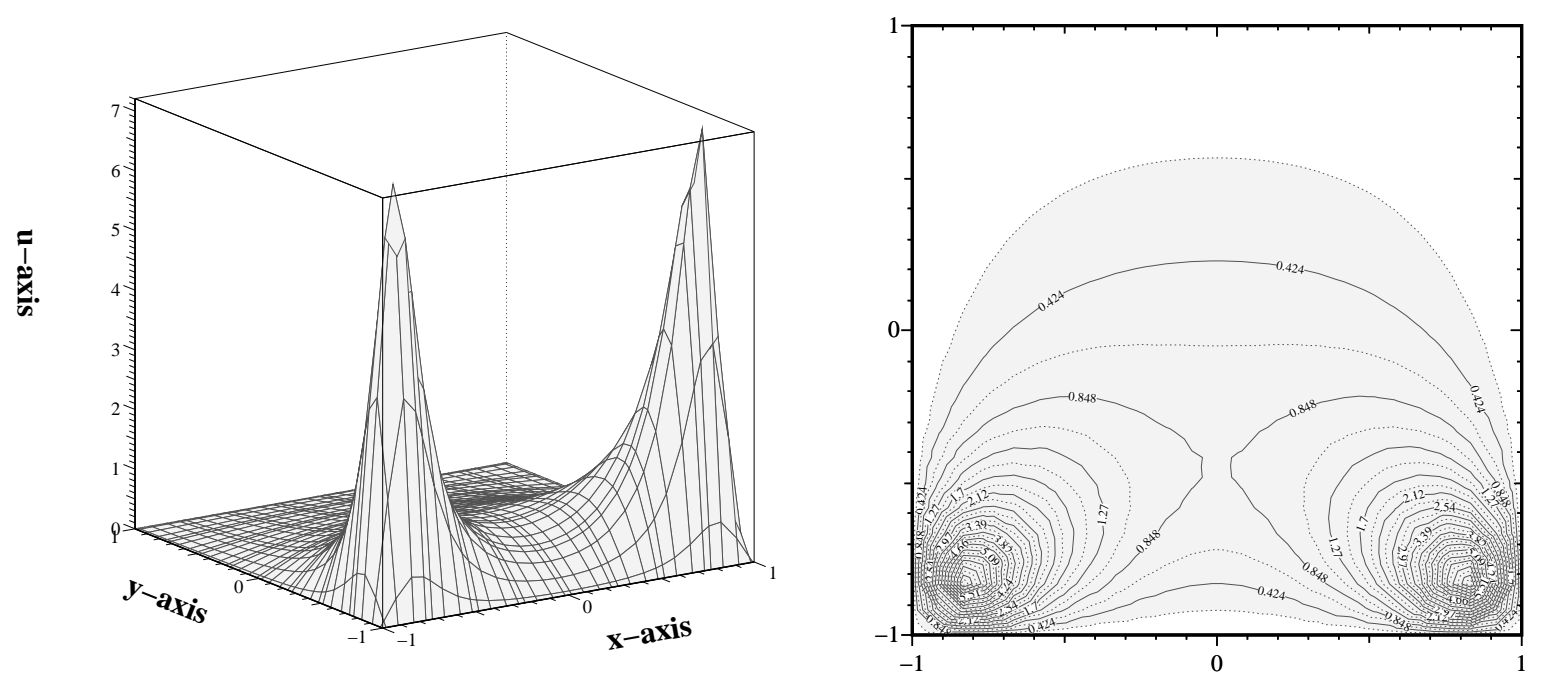

Figure 19: A solution to (3.2) with $q=9, J=86.34$
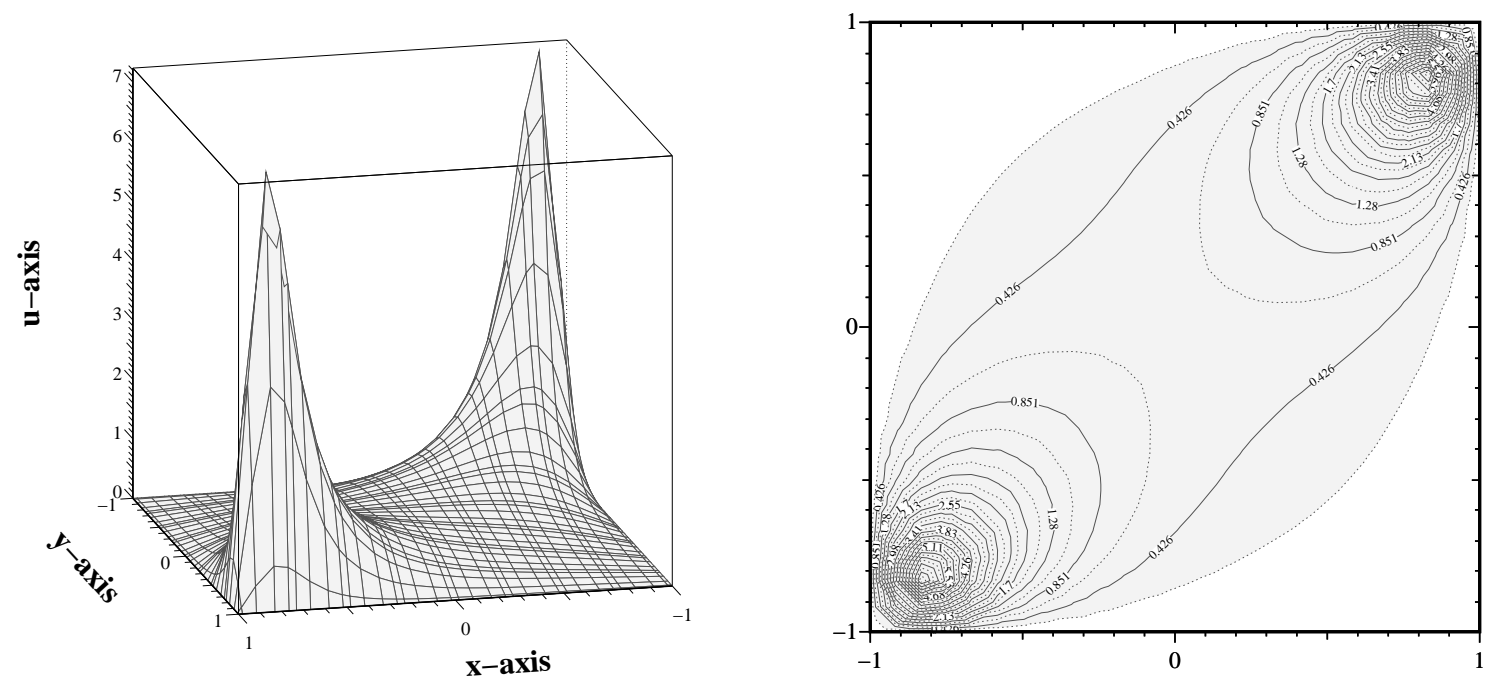

Figure 20: A solution to (3.2) with $q=9, J=86.61$
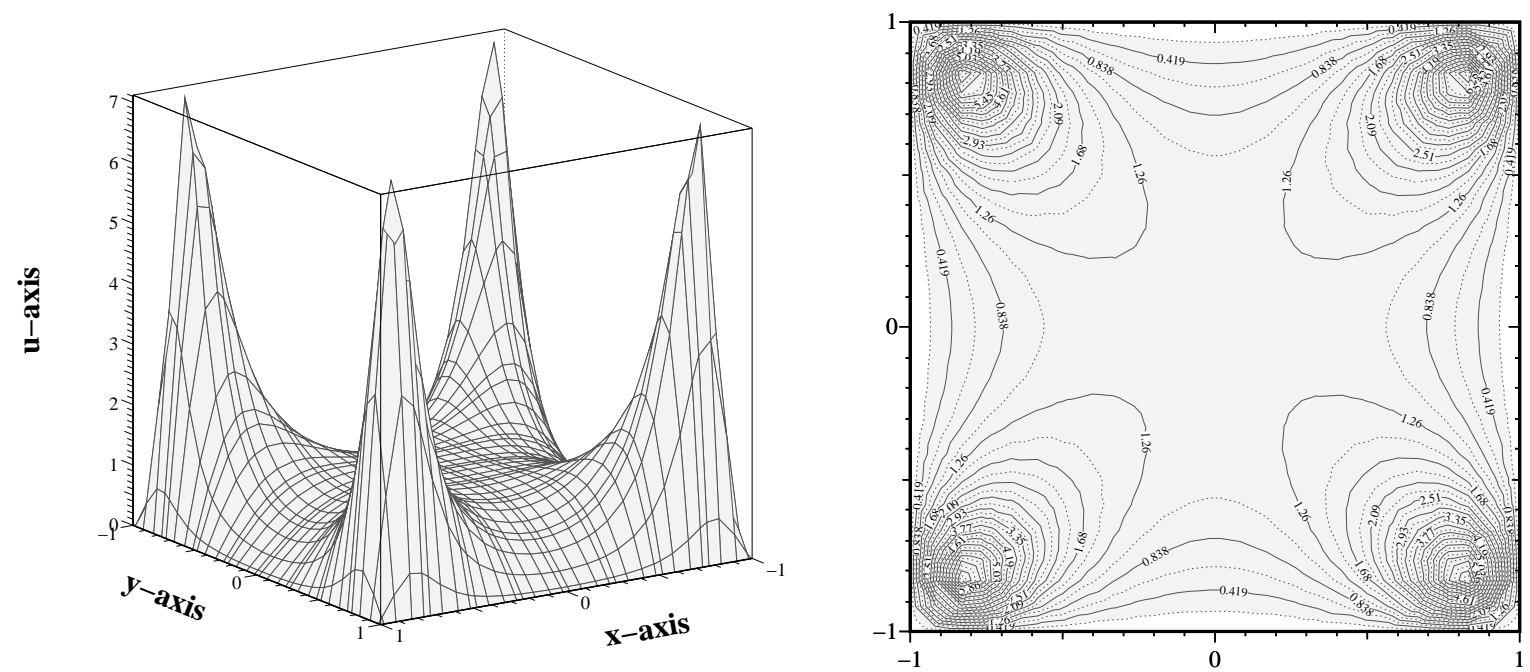

Figure 21: A solution to (3.2) with $q=9, J=171.0$ 

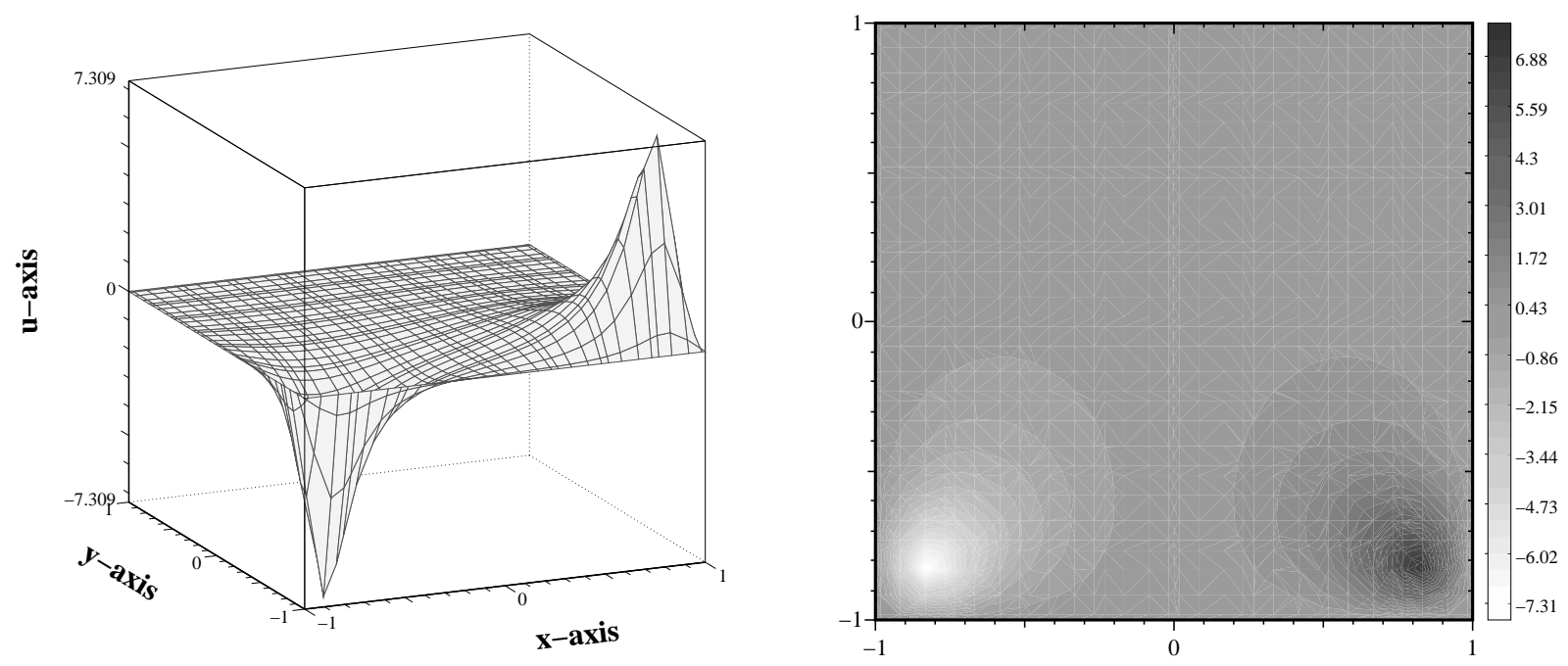

Figure 22: A solution to (3.2) with $q=9, J=87.42$,
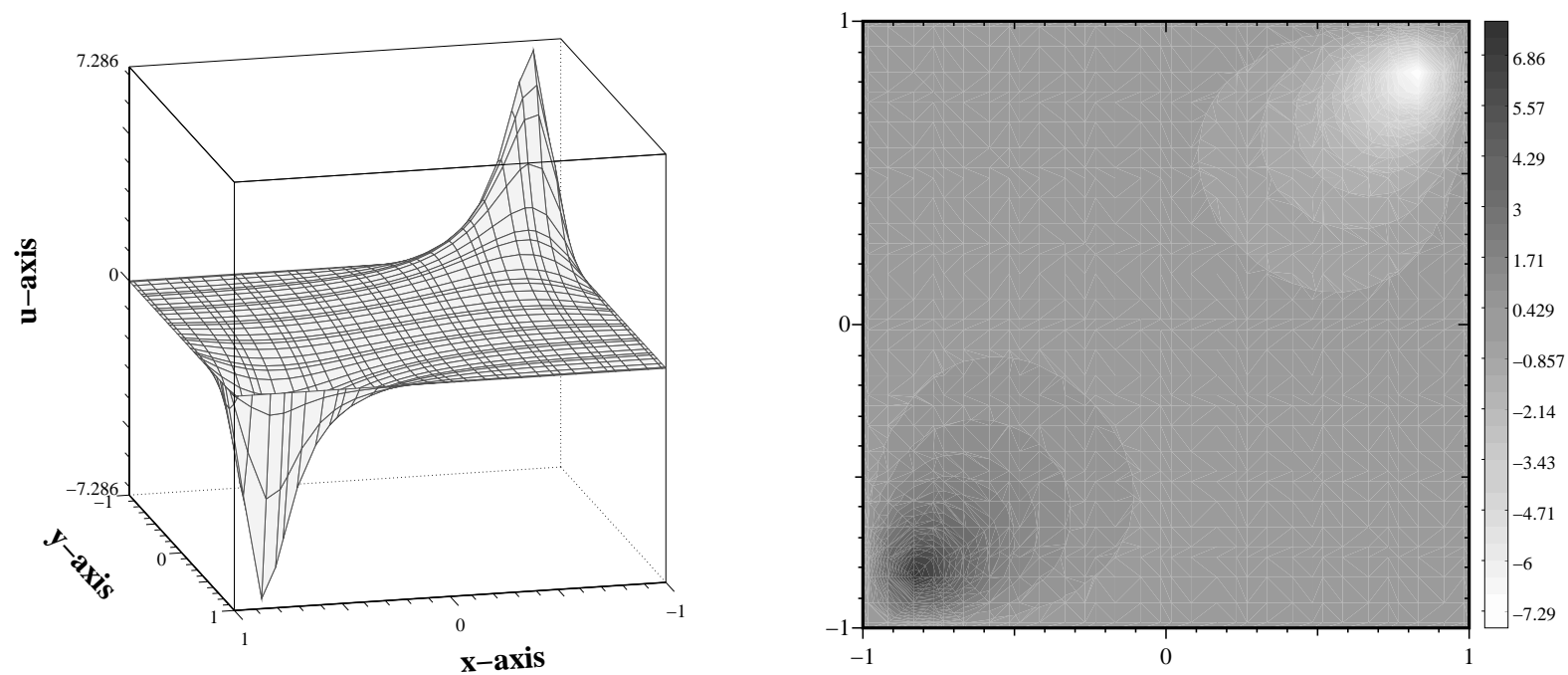

Figure 23: A solution to (3.2) with $q=9, J=87.15$,
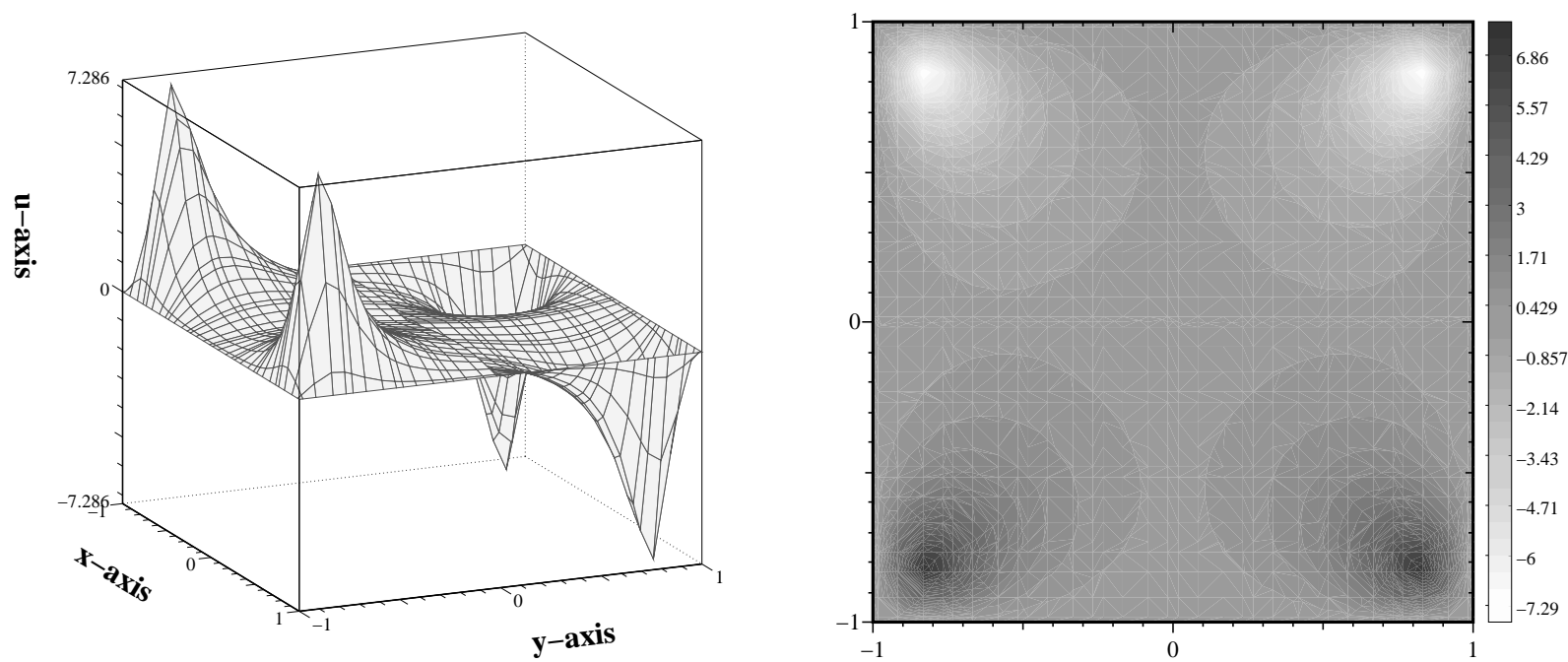

Figure 24: A solution to (3.2) with $q=9, J=174.3$ 

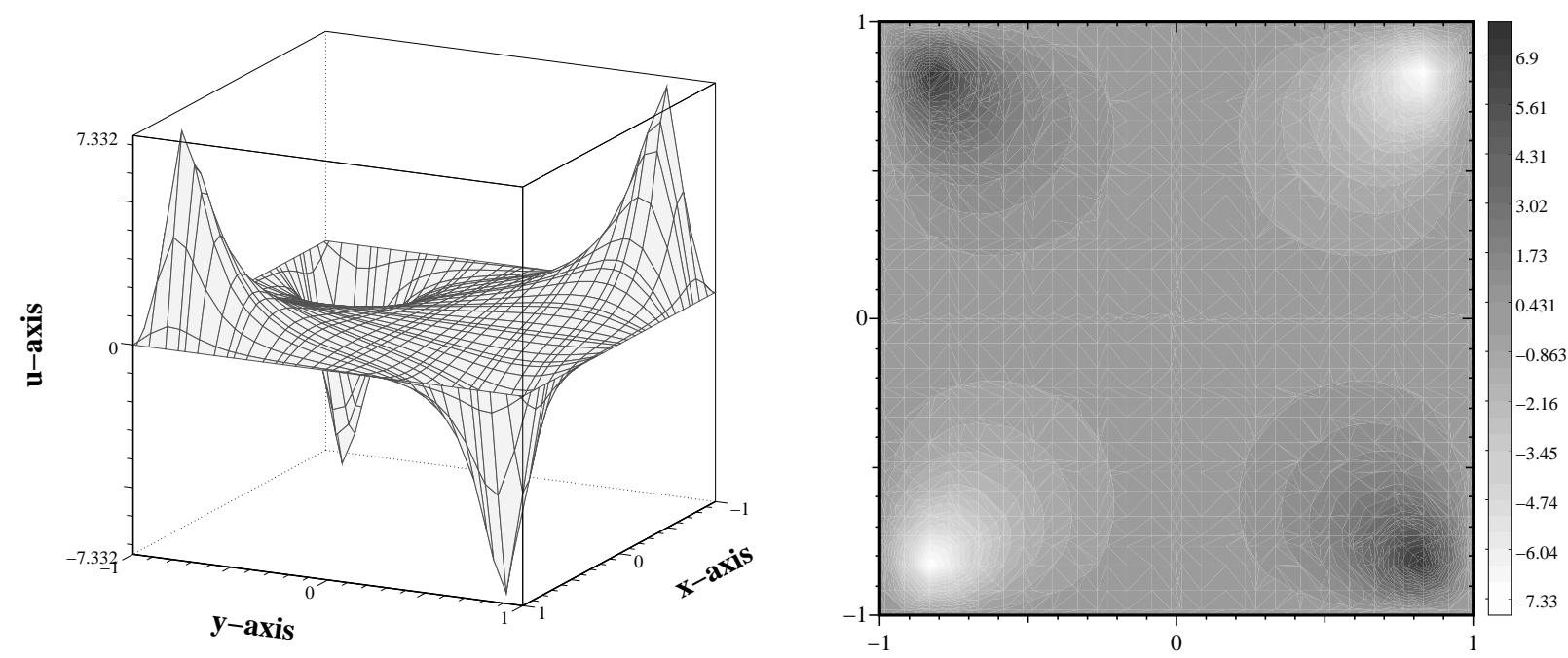

Figure 25: A solution to (3.2) with $q=9, J=175.4$
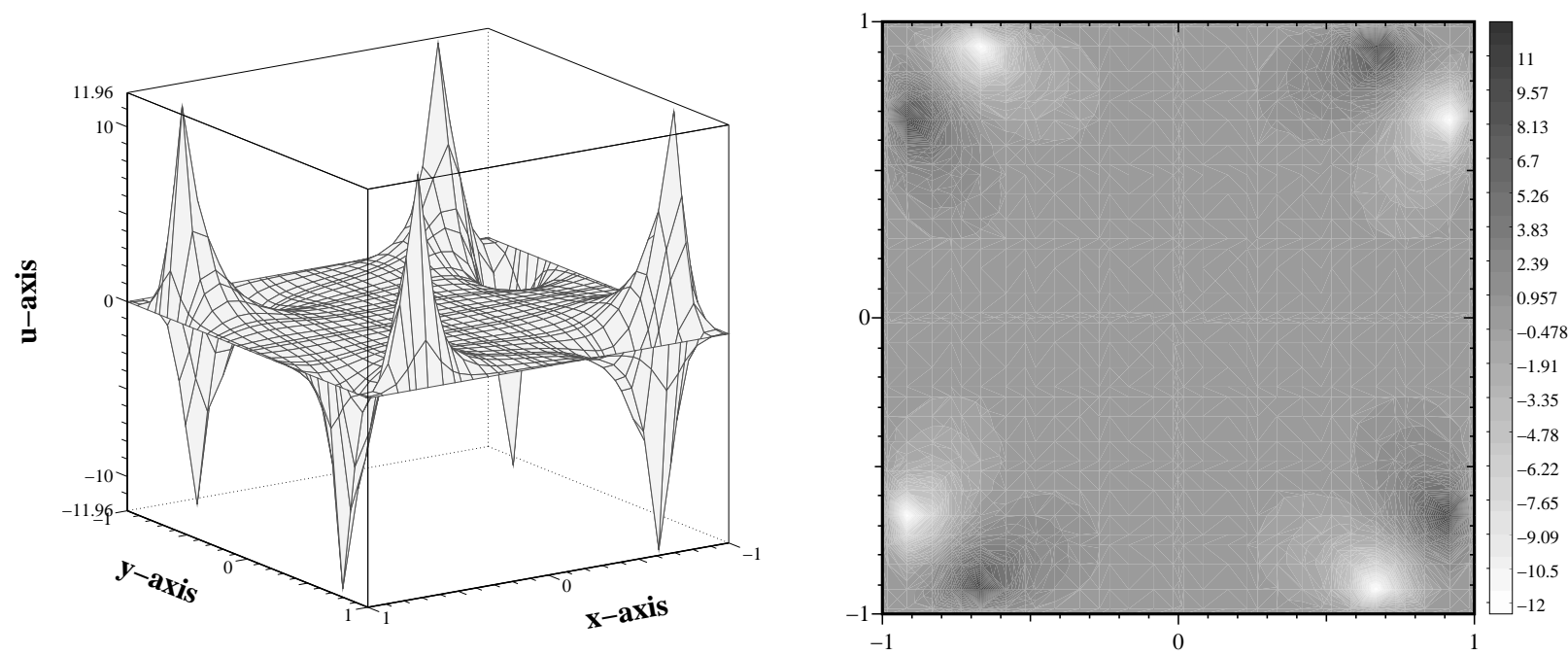

Figure 26: A solution to (3.2) with $q=9, J=1027$.
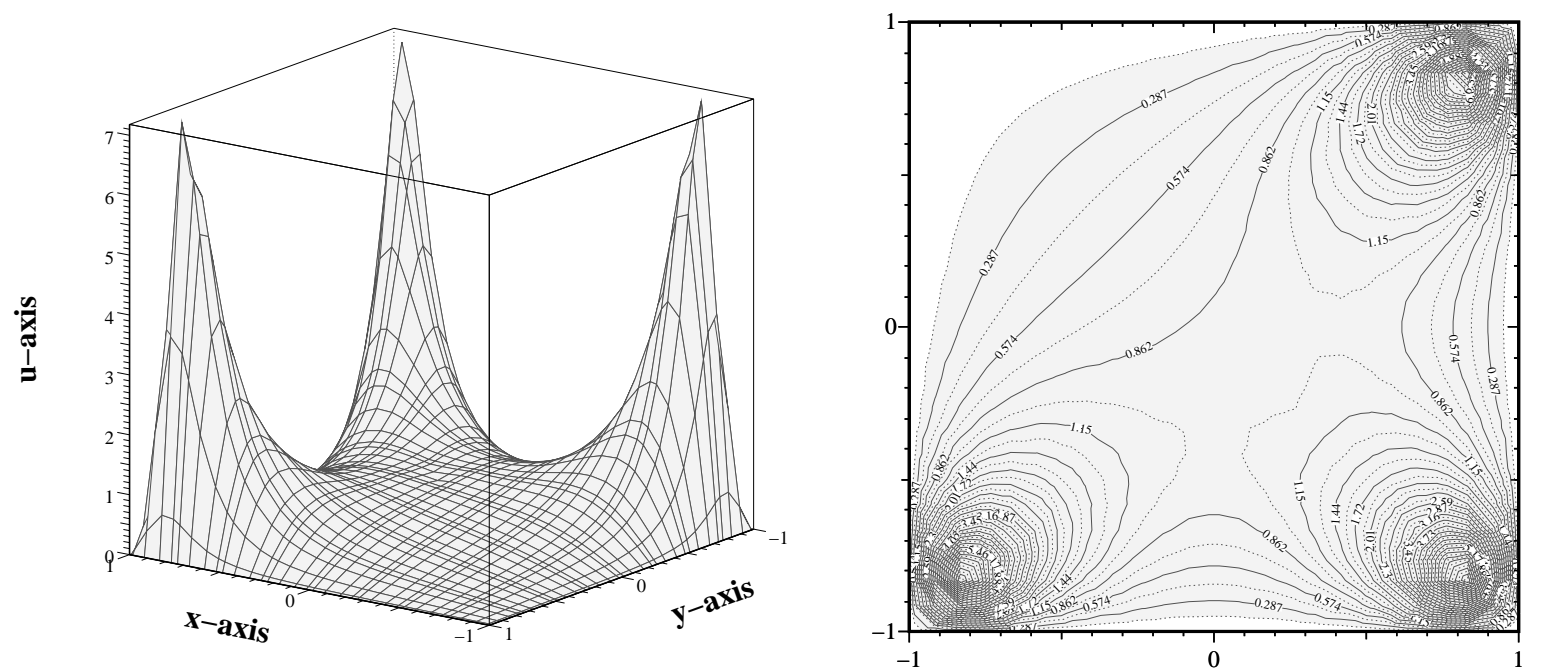

Figure 27: A solution to (3.2) with $q=9, J=129.1$ 


\section{References}

[1] A. Ambrosetti and P. Rabinowitz, Dual variational methods in critical point theory and applications, J. Funct. Anal. 14(1973), 349-381.

[2] T. Bartsch and Z.-Q. Wang, On the existence of sign-changing solutions for semilinear Dirichlet problems, Topol. Methods Nonlinear Anal., 7 (1996), 115-131.

[3] H. Brezis and L. Nirenberg, Remarks on Finding Critical Points, Comm. Pure Appl. Math., Vol. XLIV, (1991), 939-963.

[4] J. Byeon and Z.-Q. Wang, On the Hénon equation : asymptotic profile of ground states, preprint.

[5] F. Catrina and Z.-Q. Wang, Nonlinear elliptic equations on expanding symmetric domains, Journal of Differential Equations, 156(1999), 153 - 181.

[6] K.C. Chang, Infinite Dimensional Morse Theory and Multiple Solution Problems, Birkhäuser, Boston, 1993.

[7] Y. S. Choi and P. J. McKenna, A mountain pass method for the numerical solution of semilinear elliptic problems, Nonlinear Analysis, Theory, Methods and Applications, 20(1993), 417-437.

[8] D. Costa, Z. Ding and J. Neuberger, A numerical investigation of sign-changing solutions to superlinear elliptic equations on symmetric domains, J. Comp. Appl. Math., 131(2001), 299-319.

[9] W.Y. Ding and W.M. Ni, On the existence of positive entire solutions of a semilinear elliptic equation, Arch. Rational Mech. Anal., 91(1986) 238-308.

[10] Z. Ding, D. Costa and G. Chen, A high linking method for sign changing solutions for semilinear elliptic equations, Nonlinear Analysis, 38(1999) 151-172.

[11] S. Li and Z.-Q. Wang, Ljusternik-Schnirelman theory in partially orderes Hilbert spaces, AMS Transaction 354(2002), 3207-3227.

[12] Y. Li and J. Zhou, A minimax method for finding multiple critical points and its applications to semilinear PDE, SIAM Sci. Comp. 23(2001), 840-865

[13] Y. Li and J. Zhou, Convergence results of a minimax method for finding multiple critical points, SIAM Sci. Comp. to appear. 
[14] Y. Li and J. Zhou, Local characterizations of saddle points and their Morse indices, in "Control Nonlinear Distributed Parameter Systems", G. Chen, I. Lasiecka and J. Zhou, eds., Chap. 11, Lecture Notes in Pure \& Appl. Math., Vol. 218, Marcel Dekker, New York, 2001, 233-251.

[15] Z. Liu and J. Sun, Invariant sets of descending flow in critical point theory with applications to nonlinear differential equations, J. Diff. Equ., 172(2001), 257-299.

[16] J. Mawhin and M. Willem, Critical Point Theory and Hamiltonian Systems, SpringerVerlag, New York, 1989.

[17] Z. Nehari, On a class of nonlinear second-order differential equations, Trans. Amer. Math. Soc., 95(1960), 101-123.

[18] W.M. Ni, Some Aspects of Semilinear Elliptic Equations, Dept. of Math. National Tsing Hua Univ., Hsinchu, Taiwan, Rep. of China, 1987.

[19] W.M. Ni, Recent progress in semilinear elliptic equations, in RIMS Kokyuroku 679, Kyoto University, Kyoto, Japan, 1989, 1-39.

[20] P. Rabinowitz, Minimax Method in Critical Point Theory with Applications to Differential Equations, CBMS Reg. Conf. Series in Math., No. 65, AMS, Providence, 1986.

[21] M. Schechter, Linking Methods in Critical Point Theory, Birkhauser, Boston, 1999.

[22] M. Struwe, Variational Methods, Springer, 1996.

[23] Z.-Q. Wang, On a superlinear elliptic equation, Ann. Inst. Henri Poincare, 8(1991), 43-57.

[24] M. Willem, Minimax Theorems, Birkhauser, Boston, 1996.

[25] J. Zhou, Local Instability Indices of Saddle Points by a Local Minimax Method, submitted. 